\title{
ABOVE AND BELOW SUBGROUPS OF A LATTICE-ORDERED GROUP
}

\author{
RICHARD N. BALL, ${ }^{1}$ PAUL CONRAD AND MICHAEL DARNEL
}

\begin{abstract}
In an $l$-group $G$, this paper defines an $l$-subgroup $A$ to be above an $l$-subgroup $B$ (or $B$ to be below $A$ ) if for every integer $n, a \in A$, and $b \in B$, $n(|a| \wedge|b|) \leqslant|a|$. It is shown that for every $l$-subgroup $A$, there exists an $l$-subgroup $B$ maximal below $A$ which is closed, convex, and, if the $l$-group $G$ is normal-valued, unique, and that for every $l$-subgroup $B$ there exists an $l$-subgroup $A$ maximal above $B$ which is saturated: if $0=x \wedge y$ and $x+y \in A$, then $x \in A$.

Given $l$-groups $A$ and $B$, it is shown that every lattice ordering of the splitting extension $G=A \times B$, which extends the lattice orders of $A$ and $B$ and makes $A$ lie above $B$, is uniquely determined by a lattice homomorphism $\pi$ from the lattice of principal convex $l$-subgroups of $A$ into the cardinal summands of $B$. This extension is sufficiently general to encompass the cardinal sum of two l-groups, the lex extension of an $l$-group by an o-group, and the permutation wreath product of two l-groups.

Finally, a characterization is given of those abelian l-groups $G$ that split off below: whenever $G$ is a convex $l$-subgroup of an $l$-group $H, H$ is then a splitting extension of $G$ by $A$ for any $l$-subgroup $A$ maximal above $G$ in $H$.
\end{abstract}

0. Introduction. Though the groups are not in general abelian, the notation is additive with the lattice operations taking priority: $a+b \wedge c$ is $a+(b \wedge c)$, $-b \wedge c$ is $(-b) \vee(-c)$, but $n a \wedge b$ is $(n a) \wedge b . g^{+}, g^{-}$, and $|g|$ denote as usual $g \vee 0,-g \vee 0$, and $g \vee(-g)$, respectively. $g$ is a component of $h$ if $|g| \wedge|h-g|=0$; we remind the reader that components commute and that $x+y=x \vee y$ whenever $x$ and $y$ are disjoint, that is, whenever $x \wedge y=0 . \mathscr{C}(G), \mathscr{C} p(G), \mathscr{K}(G), \mathscr{S}(G)$, and $\mathscr{P}(G)$ denote respectively the lattices of convex $l$-subgroups, principal convex $l$-subgroups, closed convex $l$-subgroups, cardinal summands, and polars. (The reader unfamiliar with these basic concepts can find them in [6].) $\mathscr{P}(G)$ is a complete Boolean algebra with $\mathscr{S}(G)$ as a subalgebra. We write $A \leqslant B$ to indicate that $A$ is an $l$-subgroup of $G$, and $G(a)$ for $\{g \in G:|g| \leqslant n|a|$ for sorne integer $n\}$, the principal convex $l$-subgroup of $G$ generated by $a$. If $A$ is any subset of $G$ we use $\langle A\rangle$ to designate the $l$-subgroup generated by $A$. Note that the elements of $\langle A\rangle$ have the form $\bigvee_{i=1}^{n} \wedge_{j=1}^{m} x_{i j}$, where the $x_{i j}$ 's are from the subgroup generated by $A$. Finally, $b \ll a$ means that $n|b| \leqslant|a|$ for all integers $n$.

Received by the editors October 23, 1984 and, in revised form, August 8, 1985.

1980 Mathematics Subject Classification (1985 Revision). Primary 06F15; Secondary 20E22.

${ }^{1}$ This work was done when the first author was a visiting associate professor of mathematics at the University of Kansas. He would like to express his appreciation for the hospitality he enjoyed there. 
A subgroup $P$ of $G$ is regular if it is maximal in $\mathscr{C}(G)$ with respect to omitting some element $g$, and we say that $P$ is a value of $g$ in this case. Such a subgroup is prime inasmuch as it contains at least one element from each disjoint pair. A set $\Gamma$ of regular subgroups is plenary provided that $\cap \Gamma=0$ and that $P \in \Gamma$ and $P \subseteq Q$ for a regular subgroup $Q$ imply $Q \in \Gamma$. Such a set forms a root system in the inclusion order; that is, a partially ordered set such that for any $\gamma \in \Gamma$ the set $\{\delta \in \Gamma: \delta \geqslant \gamma\}$ is a chain. Most often we view $\Gamma$ as an index set and use small Greek letters to indicate its elements; this abuse of notation allows us to write the corresponding regular prime as $G_{\gamma}$ for $\gamma \in \Gamma$ and to use $G^{\gamma}=\bigcap\left\{G_{\delta}: \delta \in \Gamma\right.$ and $\left.G_{\gamma} \subset G_{\delta}\right\}$ for its cover. Note that $G^{\gamma}$ is prime but not in general regular, and so $G^{\gamma}$ need not correspond to a member of $\Gamma$. $G$ is said to be normal-valued if each $G_{\gamma}$ is normal in $G^{\gamma}$. If $B$ is any subset of $G$, then $\Gamma(B)$ designates $\left\{\gamma \in \Gamma: G_{\gamma}\right.$ is a value of some $b \in B$ \}. An element $g$ is special if $\Gamma(g)$ is a singleton, and in this case we term its value special also.

Given $l$-groups $A$ and $B$ their cardinal sum $A \boxplus B$ is their group direct sum with cardinal order: $a+b \geqslant 0$ if and only if $a \geqslant 0$ and $b \geqslant 0$. If $\left\{G_{\lambda}: \lambda \in \Lambda\right\}$ is a set of l-groups, then their cardinal product (it should be called the large sum but is not) is $\Pi G_{\lambda}=\left\{f: \Lambda \rightarrow \bigcup G_{\lambda}: f(\lambda) \in G_{\lambda}\right.$ for all $\left.\lambda \in \Lambda\right\}$ with componentwise group and lattice operations. The cardinal sum (or small sum) is $\left\{f \in \Pi G_{\lambda}:\{\lambda \in \Lambda: f(\lambda) \neq 0\}\right.$ is finite $\}$. Now suppose $\Gamma$ is any root system and $S$ any subgroup of the real numbers R. $V(\Gamma, S)$ designates $\{f: \Lambda \rightarrow S: \operatorname{supp}(f)$ admits no infinite ascending chains $\}$, where $\operatorname{supp}(f)=\{\lambda \in \Lambda: f(\lambda) \neq 0\}$. The group operations in $V(\Gamma, S)$ are componentwise and the order is determined by declaring $f \geqslant 0$ if and only if $f(\lambda)>0$ for all maximal elements $\lambda$ of $\operatorname{supp}(f)$. The l-groups $V(\Gamma, S)$ are universal for abelian l-groups [12]. $\Sigma(\Gamma, S)=\{g \in V(\Gamma, S)$ : $\operatorname{supp}(g)$ is finite $\}$.

Now let $\mathscr{B}$ be a Boolean algebra. $1_{\mathscr{B}}$ will denote the greatest element of $\mathscr{B}$ and $0_{\mathscr{B}}$ the least element. If $\mathscr{B}=\mathscr{P}(G)$ for an $l$-group $G$, then, $1_{\mathscr{D}}=G$ and $0_{\mathscr{B}}=0$.

1. Above and below subgroups. In this section we characterize in various ways what it means for an $l$-subgroup $A$ to lie above another $l$-subgroup $B$ in an $l$-group $G$. We show that for any $l$-subgroup $A$ there is an $l$-subgroup $B$ maximal below $A$ which is convex, closed, and unique in the normal valued case, and that for any $l$-subgroup $B$ there is an $l$-subgroup $A$ maximal above $B$ which is closed and saturated. These results are fundamental for the rest of the paper.

Proposition 1.1. The following are equivalent for elements $a$ and $b$ in $G$.

(a) $|a| \wedge|b| \ll|a|$.

(b) $n|b| \wedge|a|$ is a component of $n|b|$ for all positive integers $n$.

(c) There is no prime $P$ and no integer $n$ for which $P<P+|a|<P+n b$.

If these conditions hold, then $G(b)=(G(b) \cap G(a)) \boxplus\left(G(b) \cap a^{\perp}\right)$, and we designate the projection l-homomorphisms $\nu a: G(b) \rightarrow G(a)$ and $\mu a: G(b) \rightarrow a^{\perp}$; that is, $x \nu a \in G(a), x \mu a \in a^{\perp}$, and $x=x \nu a+x \mu a$ for all $x \in G(b)$. In particular, $x \nu a=x^{+} \wedge a^{+}+x^{+} \wedge a^{-}-x^{-} \wedge a^{+}-x^{-} \wedge a^{-}$for all $x \in G(b)$.

Proof. Suppose $0 \leqslant a \wedge b \ll a$ and let $b_{0}=b \wedge a$ and $b_{1}=b-b_{0}=(b-a)^{+}$. We claim $b_{0} \wedge b_{1}=0$, for which it is sufficient to show $b_{1} \wedge a=0$. For if $P$ is any 
prime such that $P+b_{1} \wedge a>P$, then $P+b>P+a>P$, whence $P+a+b \geqslant$ $P+2 a>P+a$ and $P+2 b \geqslant P+b+a \geqslant P+b>P+a$, meaning

$$
P+2 a \wedge(a+b) \wedge(b+a) \wedge 2 b=P+2(a \wedge b)>P+a,
$$

contradicting $a \wedge b \ll a$. The claim shows that $b_{0}$ and $b_{1}$ are complementary components of $b$, hence $n b_{0}$ and $n b_{1}$ are complementary components of $n b$ for any positive integer $n$. But $n b_{1} \in a^{\perp}$, hence $n b \wedge a=n b_{0} \wedge a=n b_{0}$ since $b_{0} \ll a$. This shows that (a) implies (b). Now suppose (b) holds and that $P$ is a prime for which $P<P+|a|$. Since $n|b|=n|b| \wedge|a|+z$ for some $z \in a^{\perp} \subseteq P, P+n b \leqslant P$ $+n|b|=P+n|b| \wedge|a| \leqslant P+|a|$, which establishes (c). Finally suppose $|a| \wedge|b|$ $\ll|a|$ fails—say $n(|a| \wedge|b|) \nless|a|$ for some positive integer $n$. Then there must be some prime $P$ for which $P+n(|a| \wedge|b|)>P+|a|$. Observe that

$$
P<P+|a| \wedge|b| \leqslant P+|a|<P+n(|a| \wedge|b|) \leqslant P+n|b|=P+n b \vee(-n) b .
$$

This proves that (c) implies (a).

Consider arbitrary $a$ and $b$ satisfying the conditions, and fix $x \in G(b)$. Then there is some integer $n$ for which $|x| \leqslant n|b|$, and the argument that (a) implies (b) above shows that

$$
k\left(x^{+} \wedge|a|\right) \vee k\left(x^{-} \wedge|a|\right) \leqslant k(|x| \wedge|a|) \leqslant k(n|b| \wedge|a|)=k n(|b| \wedge|a|) \leqslant|a|
$$

for all positive integers $k$. That is, the properties above hold when $b$ is replaced by either $x^{+}$or $x^{-}$. Therefore there are unique elements $p$ and $q$ in $a^{\perp}$ such that $x^{+}=x^{+} \wedge|a|+p=x^{+} \wedge a^{+}+x^{+} \wedge a^{-}+p$ and $x^{-}=x^{-} \wedge|a|+q=x^{-} \wedge a^{+}+x^{-}$ $\wedge a^{-}+q$. By substituting these expressions into $x=x^{+}-x^{-}$one obtains $x=x \nu a$ $+(p+q) \in(G(b) \cap G(a)) \boxplus\left(G(b) \cap a^{\perp}\right)$.

If $a$ and $b$ satisfy the conditions of Proposition 1.1 we shall say that $a$ lies above $b$ or that $b$ lies below $a$. More generally, we shall say that a subset $A \subseteq G$ lies above a subset $B \subseteq G$, or that $B$ lies below $A$, if each element of $A$ lies above each element of $B$.

Proposition 1.2. Suppose $G$ is a normal-valued l-group having positive elements a and $b$ and plenary set $\Delta$ of regular primes. Then $b \ll a$ if and only if $a \notin G^{\delta}$ for all $\delta \in \Delta(b)$, and $b$ lies below $a$ if and only if $b \in G_{\delta}$ for all $\delta \in \Delta(a)$.

Proof. If $b \ll a$ and $\delta \in \Delta(b)$, then $G_{\delta}+n b<G_{\delta}+a$ for all positive integers $n$, hence $a \notin\left\{g \in G: G_{\delta}+|g| \leqslant G_{\delta}+n b\right.$ for some $\left.n\right\}=G^{\delta}$. Conversely, if it is not true that $b \ll a$ then there is some positive integer $n$ and $\delta \in \Delta$ with $G_{\delta}+n b>$ $G_{\delta}+a$. Clearly $a$ lies in the cover of the unique value of $b$ containing $G_{\delta}$. Now suppose $b$ is below $a$ and consider $\delta \in \Delta(a)$. Then $a \in G^{\delta}$ implies $a \wedge b \in G_{\delta}$, so $G_{\delta}=G+a \wedge b=G+b$, meaning $b \in G_{\delta}$. If $b$ is not below $a$ then there is some $\delta \in \Delta(a \wedge b)$ for which $a \in G^{\delta}$. But then $a \notin G_{\delta}$ implies $\delta \in \Delta(a)$, and $b \notin G_{\delta}$.

The failure of Proposition 1.2 in $A(\mathbf{R})$, the $l$-group of order-preserving automorphisms of the real numbers $\mathbf{R}$, shows that the hypothesis of normal-valuedness cannot be omitted from Proposition 1.2, or indeed from the other results of this section in which it appears. 
Proposition 1.3. For each positive element $a$ in the normal valued l-group $G$, $D=\{b:|b| \ll a\}$ is the unique largest subgroup of $G$ such that $D \leqslant a$, and $D \in$ $\mathscr{K}(G)$. Furthermore, $C=a^{\perp} \boxplus D=\bigcap\left\{G_{\delta}: \delta \in \Delta(a)\right\}=\{b: b$ lies below $a\}$ is the unique largest subgroup of $G$ which lies below $a$, and $C \in \mathscr{K}(G)$.

Proof. Let $\Delta$ be any plenary set of regular primes of $G$. Consider $0<b_{1}, b_{2} \in D$ and $\delta \in \Delta\left(b_{1}+b_{2}\right)$. Since $\Delta\left(b_{1}+b_{2}\right) \subseteq \Delta\left(b_{1}\right)+\Delta\left(b_{2}\right), a \notin G^{\delta}$ by Proposition 1.2 , hence $b_{1}+b_{2} \ll a$. It follows that $D \in \mathscr{C}(G)$, and is therefore clearly maximal with respect to $D \leqslant a$. To show $D$ closed suppose $\bigvee E=e$, where $E=\{b \in D$ : $b \leqslant e\}$. By Lemma 1.17 of [4], ne $=\bigvee\{n b: b \in E\}$ for each positive integer $n$. Now $\{n b: b \in E\} \subseteq D \leqslant a$, so $n e \leqslant a$, proving $a \gg e \in D \in \mathscr{K}(G)$.

Propositions 1.1 and 1.2 establish $\bigcap\left\{G_{\delta}: \delta \in \Delta(a)\right\}=\{b: b$ lies below $a\} \subseteq a^{\perp}$ $\boxplus D$. But for any $\delta \in \Delta(a), a \notin G_{\delta}$ implies $a^{\perp} \subseteq G_{\delta}$, and $D \subseteq G_{\delta}$ by Proposition 1.2. This shows all those sets are equal. The closure of $C$ is a straightforward consequence of the closure of $a^{\perp}$ and of $D$.

Proposition 1.4. The following are equivalent for subgroups $A$ and $B$ of $G$.

(a) $A$ lies above $B$.

(b) bva is a component of $b$ for each $b \in B$ and $a \in A$.

(c) There are no elements $a \in A$ and $b \in B$ and no prime $P$ satisfying $P<P+a<$ $P+b$. That is, $a^{+} \wedge(b-a)^{+}=0$ for all $a \in A$ and $b \in B$.

If $G$ is normal-valued with plenary set $\Delta$ of regular primes, then the conditions above are equivalent to the condition that $\delta_{1} \neq \delta_{2}$ for all $\delta_{1} \in \Delta(B)$ and $\delta_{2} \in \Delta(A)$, or that $B \subseteq \bigcap\left\{G_{\delta}: \delta \in \Delta(A)\right\}$. If , in addition, $B$ is a convex l-subgroup, then the conditions above are equivalent to $\Delta(A) \cap \Delta(B)=\varnothing$. Under any circumstances, if $A$ is a convex l-subgroup, then the conditions above are equivalent to $B \subseteq A^{\perp}$.

Proof. The equivalence of conditions (a), (b), and (c) results from Proposition 1.1, the second assertion is a consequence of Proposition 1.3, and the second assertion readily implies the third. This last observation is also straightforward.

Corollary 1.5. If subgroup $A$ lies above subgroup $B$ of $G$, then $A \cap B=0$. B lies both above and below $A$ if and only if $B \subseteq A^{\perp}$.

Proof. Suppose $B$ lies both above and below $A$, and consider $a \in A, b \in B$. Then $|a| \wedge|b| \ll|a|$ and $|a| \wedge|b| \ll|b|$, meaning $|a| \wedge|b|=0$ or $B \subseteq A^{\perp}$.

Suppose $C$ is a subgroup of $G$. A subgroup $B$ which is maximal among those subgroups lying below $C$ is said to be maximal below $C$, or simply maximal below, and likewise a subgroup $A$ which is maximal among those subgroups lying above $C$ is said to be maximal above $C$, or simply maximal above. $C$ is said to be saturated if $C$ contains each component of each of its elements. Observe that a saturated subgroup $C$ is a sublattice since $c \vee 0$ is a component of each $c \in C$.

TheOREM 1.6. Suppose subgroup $A$ lies above subgroup $B$ in $G$. Then $A$ is contained in some subgroup maximal above $B$, and $B$ is contained in some subgroup maximal below $A$. If $G$ is normal-valued, then the unique subgroup maximal below $A$ is $\cap\left\{G_{\delta}\right.$ : $\delta \in \Delta(A)\}$. In any case a maximal below subgroup is a closed convex l-subgroup and a maximal above subgroup is a saturated and closed l-subgroup. 
Proof. The first assertion is a consequence of Zorn's Lemma, and the second assertion has already been established. Suppose now that $B$ is maximal below $A$ in G. $B$ must be a sublattice, for if not then there exist elements $a \in A$ and $t \in\langle B\rangle$ and prime $P$ such that $P<P+|a|<P+t$. But $t=\vee_{I} \wedge_{J} b_{i_{j}}$ for some $b_{i_{j}} \in B$ and finite $I$ and $J$, hence $P+t=P+b_{i,}$ for some $i \in I$ and $j \in J$. But then $P<P+$ $|a|<P+b_{i}$, contradicting the assumption that $B$ lies below $A$ and showing $B$ to be a sublattice. To show $B$ convex consider an arbitrary $t$ such that $b_{1} \leqslant t \leqslant b_{2}$ for $b_{1}$, $b_{2} \in B$. Then $|t| \leqslant\left|b_{1}\right| \vee\left|b_{2}\right| \in B$, hence $t$ lies below $A$. Since $\left\{t: b_{1} \leqslant t \leqslant b_{2}\right.$, $\left.b_{i} \in B\right\}$ is a subgroup containing $B, t \in B$. To show $B$ closed consider $z=\vee S$, where $S=\left\{b \in B^{+}: b \leqslant z\right\}$, and let $B^{\prime}$ be the group generated by $B \cup\{z\}$. We claim $B^{\prime}$ lies below $A$, for if not then by Proposition 1.4(c) $|a| \wedge(t-|a|)^{+} \neq 0$ for some $t \in B^{\prime}$. Now $t$ has the form $\sum_{i=1}^{n}\left(b_{i}+\varepsilon_{i} z\right)$ where $b_{i} \in B$ and $\varepsilon_{i}$ is \pm 1 . By replacing $t$ by $\sum_{i=1}^{n}\left(b_{i}+y_{i}\right)$, where $y_{i} \in S$ if $\varepsilon_{i}=-1$ and $y_{i}=z$ if $\varepsilon_{i}= \pm 1$, we may assume $t=\sum_{i=1}^{n}\left(b_{i}+z\right)$. Now $t=\bigvee U$, where $U=\left\{\sum_{i=1}^{n}\left(b_{i}+s\right): s \in S\right\}$, hence $|a| \wedge(t-|a|)^{+}=\vee\left\{|a| \wedge(u-|a|)^{+}: u \in U\right\}$, so there is some $u \in U$ for which $|a| \wedge(u-|a|)^{+}>0$. Since $u \in B$, this contradicts the assumption that $B$ lies below $A$. We can only conclude that $B$ is closed.

Suppose that $A$ is maximal above $B$. To prove $A$ saturated consider the element $a \in A$ with component $z$, and let $A^{\prime}$ be the group generated by $A \cup\{z\}$. To prove that $A^{\prime}$ lies above $B$ it is enough to show that for each $t \in A^{\prime}$ and prime $P$ there is some $a_{0} \in A$ with $P+t=P+a_{0}$. Since $t$ has the form $\sum_{i=1}^{n}\left(a_{i}+\varepsilon_{i} z\right)$, where $a_{i} \in A$ and $\varepsilon_{i}= \pm 1$, we induct on $n$. Consider the case when $n=1$ and $\varepsilon_{i}=1$. Because the prime $-a_{1}+P+a_{1}$ contains at least one of the disjoint elements $|z|$ and $|a-z|, P+a_{1}+z$ is either $P+a_{1}$ or $P+a_{1}+a$. The argument for $\varepsilon_{i}=-1$ is analogous, and the induction step yields to a simple argument using the above.

It remains to show that if $A$ is maximal above $B$ then $A$ is closed. Suppose $z=\mathrm{V} S$ for $S \subseteq A$ and let $A^{\prime}$ be the group generated by $A \cup\{z\}$. For the sake of contradiction assume that there are elements $t \in A^{\prime}$ and $b \in B$ for which $t^{+} \wedge$ $(b-t)^{+}>0$. Let us say $t$ is $\sum_{i=1}^{n}\left(a_{i}+\varepsilon_{i} z\right)$ for some $a_{i} \in A$ and $\varepsilon_{i}= \pm 1$. Then $\vee S=z$ implies $\vee R=t$, where $R=\left\{\sum_{i=1}^{n}\left(a_{i}+\varepsilon_{i} y_{i}\right): y_{i} \in S\right.$ if $\varepsilon_{i}= \pm 1$ and $y_{i}=z$ if $\left.\varepsilon_{i}=-1\right\}$, and it follows that

$$
t^{+} \wedge(b-t)^{+}=\bigvee\left\{r^{+} \wedge(b-t)^{+}: r \in R\right\} .
$$

Choose a specific $r=\sum_{i=1}^{n}\left(a_{i}+\varepsilon_{i} y_{i}\right) \in R$ such that $r^{+} \wedge(b-t)^{+}>0$. Observe that $r^{+} \wedge(b-r)^{+}>0$ also. Again, $\vee S=z$ implies $r=\wedge U$, where $U=\left\{\Sigma\left(a_{i}+\varepsilon_{i} w_{i}\right)\right.$ : $w_{i}=y_{i}$ if $\varepsilon_{i}=1$ and $w_{i} \in S$ if $\left.\varepsilon_{i}=-1\right\} \subseteq A$, and it follows that

$$
r^{+} \wedge(b-r)^{+}=\bigvee\left\{r^{+} \wedge(b-u)^{+}: u \in U\right\} .
$$

Choose a specific $u=\sum_{i=1}^{n}\left(a_{i}+\varepsilon_{i} w_{i}\right) \in U$ such that $r^{+} \wedge(b-u)^{+}>0$. Then $u^{+}$ $\wedge(b-u)^{+}>0$ and $u \in A$, contradicting the assumption that $A$ lies above $B$.

If subgroup $B$ of $G$ is maximal below and $A$ is maximal above $B$, then it need not follow that $B$ is maximal below $A$. Consider $G=\mathbf{Z} \overrightarrow{\times}(\mathbf{Z} \boxplus \mathbf{Z})$, the splitting extension of $\mathbf{Z} \boxplus \mathbf{Z}$ by $\mathbf{Z}$ lexicographically ordered, where

$$
-(m ; 0,0)+(0 ; a, b)+(m ; 0,0)= \begin{cases}(0 ; a, b) & \text { if } m \text { is even, } \\ (0 ; b, a) & \text { if } m \text { is odd }\end{cases}
$$


This is a simple example of a Scrimger group [15], studied in connection with l-group varieties; it is also a simple instance of the general construction of $\S 3$ and of Theorem 3.21 in particular. Let $B=0 \overrightarrow{\times}(\mathbf{Z} \boxplus 0)$, a subgroup maximal below $\{(\mathrm{m}$; $0, n): m$ even $\}$, and let $A=\mathbf{Z} \times \overrightarrow{\times}(0 \boxplus 0)$, another subgroup maximal above $B$. The point is that $B$ is proper in $0 \overrightarrow{\times}(\mathbf{Z} \boxplus \mathbf{Z})$ which is maximal below $A$.

$G$ is projectable if $\mathscr{P}(G)=\mathscr{S}(G)$. This concept is also termed strong projectability in the literature. A subset $Z \subseteq G^{+}$is called type $\mathscr{Y}$ if

$$
\bigcap\left\{((Z-z) \vee 0)^{\perp \perp}: z \in Z\right\}=\bigcap\left\{((-z+Z) \vee 0)^{\perp \perp}: z \in Z\right\}=0_{\mathscr{P}}
$$

[1]. For example, a pairwise disjoint subset of $G^{+}$is type $\mathscr{Y} . G$ is said to be sup $\mathscr{Y}$ complete if every type $\mathscr{Y}$ subset of $G^{+}$has a supremum in $G$, and $G$ is said to be laterally complete if every pairwise disjoint subset of $G^{+}$has a supremum in $G^{+}$. The following proposition relates these concepts, and impinges on the results of $\S 6$, but is otherwise unrelated to the subject of this section.

Proposition 1.7. A projectable l-group $G$ is sup $\mathscr{Y}$ complete if and only if it is laterally complete.

Proof. Any sup $\mathscr{Y}$ complete l-group is laterally complete. Now suppose $G$ is laterally complete and projectable, and consider a type $\mathscr{Y}$ subset $Z \subseteq G^{+}$. Let $\mathscr{U}$ be $\{\mathscr{R} \subseteq \mathscr{P}(G): \mathscr{R}$ is pairwise disjoint, and for each $R \in \mathscr{R}$ there is some $z \in Z$ with $\left.R \subseteq((Z-z) \vee 0)^{\perp}\right\}$. Now $\mathscr{U}$ is closed with respect to unions of chains, and so $\mathscr{U}$ contains a maximal element $\mathscr{R}$. Since $\vee\left\{((Z-z) \vee 0)^{\perp}: z \in Z\right\}=1_{\mathscr{P}}$ it follows that $\bigvee \mathscr{R}=1_{\mathscr{P}}$. For each $R \in \mathscr{R}$ let $a_{R}$ and $z_{R}$ satisfy $z_{R} \in Z, R \subseteq\left(\left(Z-z_{R}\right) \vee 0\right)^{\perp}$, $a_{R} \in R$, and $z_{R}-a_{R} \in R^{\perp}$. Then $a=\bigvee\left\{a_{R}: R \in \mathscr{R}\right\}$ exists by the lateral completeness of $G$. We shall prove that $\mathrm{VZ}=a$, the first step being to show $Z \leqslant a$. Suppose for contradiction that $a \nless z$ for some $z \in Z$; find $T \in \mathscr{R}$ such that $T \cap\left((z-a)^{+}\right)^{\perp \perp} \neq 0_{\mathscr{P}}$, and let $P$ be some prime subgroup satisfying $P<P+t$, where $t \in T^{+}$and $t \leqslant(z-a)^{+}$. Then $P \leqslant P+a<P+z$. Now $a-a_{T} \in T^{\perp}$, for otherwise $\left\{a_{R}: R \in \mathscr{R}\right\} \leqslant-s+a<a$ for any $s \in T$ with $0<s \leqslant a-a_{T}$, so $a-z_{T}=\left(a-a_{T}\right)-\left(z_{T}-a_{T}\right) \in T^{\perp} \subseteq P$, meaning $P+z_{T}<P+z$. But $T \subseteq$ $\left(\left(Z-z_{T}\right) \vee 0\right)^{\perp}$ implies $\left(z-z_{T}\right) \vee 0 \in T^{\perp} \subseteq P$, which in turn implies $P+z \leqslant P$ $+z_{T}$. This contradiction proves $Z \leqslant a$. $V Z=a$ by Lemma 5.6 of [1] since, as has just been pointed out, $T \subseteq\left(a-z_{T}\right)^{\perp}$ for all $T \in \mathscr{R}$, and so $\bigvee\left\{(a-z)^{\perp}: z \in Z\right\}$ $=1_{\mathscr{P}}$.

If $A \subseteq G$ we say that the polars of $\mathscr{A}$ and $\mathscr{G}$ correspond whenever the intersection map is one-one from $\mathscr{P}(G)$ onto $\mathscr{P}(A)$. This is equivalent to having each nontrivial polar of $G$ intersect $A$ nontrivially.

Corollary 1.8. Suppose $A$ is a maximal above l-subgroup of $G$. If $G$ is projectable then so is $A$. If $G$ is sup $\mathscr{Y}$ complete and polars in $G$ and $A$ correspond, then $A$ is sup $\mathscr{Y}$ complete.

Proof. Suppose $G$ is projectable, $P$ is a polar of $A$ and $Q=P^{\perp \perp}$ in $G$. For $a \in A$ let $a=a_{1}+a_{2}$, where $a_{1} \in Q$ and $a_{2} \in Q^{\perp}$. Now $a_{1}$ lies in $A$ by virtue of the saturation of $A$, and so $a_{1} \in Q \cap A=P$. Next suppose $G$ to be sup $\mathscr{Y}$ complete 
and consider a type $\mathscr{Y}$ subset $Z \subseteq A^{+}$. Because polars correspond, $Z$ is also of type $\mathscr{Y}$ considered as a subset of $G$, and so has a supremum $z \in G$. $z$ must lie in $A$ by the closure of $A$.

The relation of one subgroup being maximal above another has a tidy characterization in an abelian l-group. This characterization is important for $\$ \S 5$ and 6.

Proposition 1.9. Let $G$ be an abelian l-group with plenary set $\Delta$ of regular subgroups, and let l-subgroup $A$ lie above l-subgroup $B$ of $G$. Then $A$ is maximal above $B$ if and only if for each $g \in G \backslash A$ there is some $a \in A$ and positive integer $n$ such that $n g-a$ has a value in $\Delta(\bar{B})$.

Proof. If $A$ is maximal above $B$, then it is maximal above $\bar{B}$ by Theorem 1.6, and if $g \in G \backslash A$, then by Proposition 1.4 there is an element $n g-a$ of the subgroup generated by $A \cup\{g\}$ with $\Delta(n g-a) \cap \Delta(\bar{B}) \neq \varnothing$. The converse also follows from Proposition 1.4.

Corollary 1.10. Let $G$ be an abelian l-group with maximal above subgroup $A$. Then $n g \in A$ implies $g \in A$ for any $g \in G$ and nonzero integer $n$. Therefore $A$ is divisible if $G$ is.

Proof. $A$ is maximal above $B=\bigcap\left\{G_{\delta}: \delta \in \Gamma(A)\right\}$. If $g \notin A$, then there is some $a \in A$ and positive integer $k$ for which $k g-a$ has a value in $\Gamma(B)$. But then $n(k g-a)=k(n g)-n a \in A$ has a value in $\Gamma(B)$ also, a contradiction to the fact that $A$ lies above $B$.

Corollary 1.11. Let $G$ be a divisible abelian l-group with plenary set $\Delta$ of regular subgroups, and let l-subgroup $A$ lie above l-subgroup $B$ of $G$. Then $A$ is maximal above $B$ if and only if for each $g \in G \backslash A$ there is some $a \in A$ such that $g-a$ has a value in $\Delta(\bar{B})$.

Proof. Suppose $A$ is maximal above $B$ and that $g \in G \backslash A$, and find $a_{1} \in A$ and positive integer $n$ such that $n g-a_{1}$ has value $\delta \in \Delta(\bar{B})$. Since $G$ is divisible there is an element $a \in G$ such that $n a=a_{1}$, and $a \in A$ by the previous corollary. Since $n(g-a)=n g-a_{1}, g-a$ has value $\delta$.

Corollary 1.12. A maximal above subgroup $A$ is convex if and only if $A$ is a polar if and only if $A$ is also maximal below.

Proof. If $A$ is maximal above $B$ and convex, then $A \subseteq B^{\perp}$ by Proposition 1.4, and since $B^{\perp}$ lies above $B$ by Corollary $1.5, A=B^{\perp}$ and so $A$ is a polar. But any polar is maximal below its complementary polar by Corollary 1.5. And any maximal below subgroup is convex by Theorem 1.6.

Given subgroup $B$ of $G$ let $\mathscr{A}(B)$ designate the collection of all subgroups of $G$ maximal above $B$. Note that $\mathscr{A}(B) \neq \varnothing$ since the $o$-subgroup lies above $B$ and is therefore contained in some subgroup maximal above $B$ by Theorem 1.6.

Proposition 1.13. กब $(B) \subseteq B^{\perp}$ for any subgroup $B$ of $G$. Therefore if $B$ has only one subgroup maximal above it, then this subgroup is $B^{\perp}$. G is representable if and only if $\cap \mathscr{A}(B)=B^{\perp}$ for all subgroups $B$ of $G$. 
Proof. Consider $0<a \in \cap \mathscr{A}(B)$. By Theorem 1.6 we may assume $B \in \mathscr{C}(G)$. Then for any $0<b \in B,\langle a+a \wedge b\rangle$ lies above $B$, for if $P<P+a+a \wedge b<P$ $+b_{1}$ for some $b_{1} \in B$ and prime $P$, then $P \leqslant P+a \leqslant P+a+a \wedge b<P+b_{1}$ implies $a \in P$, hence $a \wedge b \in P$, contradicting $P<P+a+a \wedge b$. Therefore there is some $A \in \mathscr{A}(B)$ such that $a+a \wedge b \in A$. But then $a \in A$ implies $a \wedge b \in A \cap$ $B=0$. This argument shows that $\cap \mathscr{A}(B) \subseteq B^{\perp}$. Now suppose $B$ is a subgroup of the representable $l$-group $G$ having plenary set $\Delta$, and consider $A \in \mathscr{A}(B)$. Because $B^{\perp}$ is normal in $G$, the subgroup generated by $B^{\perp} \cup A$ is $B^{\perp}+A$; consider an arbitrary $c+a \in B^{\perp}+A$ and $\delta \in \Delta(B)$. Since $B \nsubseteq G_{\delta}$ it follows that $B^{\perp} \subseteq G_{\delta}$, hence $G_{\delta}+(c+a)=G_{\delta}+a$, so $a \notin G^{\delta}$. This shows that $B^{\perp}+A$ lies above $B$, and by maximality that $B^{\perp} \subseteq A$. Thus $\cap \mathscr{A}(B)=B^{\perp}$. Finally, if $G$ is not representable then it is only because it contains elements $b, g>0$ for which $(g+b-g) \wedge b$ $=0$. We claim $\langle g\rangle$ lies above $\langle b\rangle$, for if $P<P+g<P+n b$ for some positive integer $n$, then $P+g-n b-g<P-g<P$, hence $P<P+(g+n b-g) \wedge b$, a contradiction. Therefore there is some $A \in \mathscr{A}\langle b\rangle$ such that $g \in A$. But $b^{\perp} \subseteq A$ would give $g+b-g \in A$, which leads to the contradiction $b \in A \cap\langle b\rangle=0$. That is, $\cap \mathscr{A}\langle b\rangle \neq b^{\perp}$.

COROLlaRY 1.14. For an l-group $G$ the following are equivalent.

(a) $G$ is archimedean.

(b) Each maximal above subgroup is convex.

(c) Each maximal above subgroup is a polar.

(d) Each subgroup $B$ has a unique subgroup maximal above it.

(e) Each closed convex l-subgroup is maximal above and below.

Proof. If $G$ is archimedean and $a, b>0$, then $a \wedge b \ll a$ if and only if $a \wedge b=0$. Thus (a) implies (b), and we have proven the equivalence of (b), (c), and (d) in the last two results. To show that (d) implies (a) suppose that $G$ is not archimedean, say $0<b \ll a$. Then $\langle a\rangle$ and $\langle a+b\rangle$ both lie above $\langle b\rangle$, and yet $a$ and $a+b$ cannot both belong to the same subgroup $A$ maximal above $\langle b\rangle$, for then $b \in\langle b\rangle \cap A=0$, a contradiction. Finally, to prove the equivalence of (a) and (e) it is enough to recall a theorem of Bigard [5]: $G$ is archimedean if and only if each closed convex $l$-subgroup is a polar.

The following example from [7] sheds light on some of the ideas we have been developing. It also nicely illustrates Corollary 1.14 by barely violating each of its conditions. Let $\Gamma=\{1,2,3, \ldots, \omega, \alpha\}$, partially ordered by declaring only that $\omega>\alpha$. Let $G=\left\{g \in V(\Gamma, \mathbf{R}): \lim _{n \rightarrow \infty} g(n)=g(\omega)\right\}$. Let $H=\{g \in G: g(\omega)=$ $0\}$. One readily checks that $G$ is a nonarchimedean $l$-group all of whose convex l-subgroups are closed. The maximal below subgroups are precisely the polars, however, and in particular the only subgroup above $H$ is (0). The polar $G_{1}=\{g \in G$ : $g(n)=0$ for all $n=1,2,3, \ldots\}$ has numerous subgroups maximal above it, no one of which is convex. Also observe that $H$ is the join in $\mathscr{K}(G)$ of the polars $E=\{g \in G: g(n)=0$ for all odd integers $n\}$ and $O=\{g \in G: g(n)=0$ for all even integers $n$ \}. Thus the maximal below subgroups do not constitute a sublattice of $\mathscr{K}(G)$. 
In some instances an $l$-subgroup $A$ will lie above an $l$-subgroup $B$ provided only that $A \cap B=0$. The remainder of this section is devoted to an investigation of some of these instances.

Lemma 1.15. Suppose $t, g \in G^{+}$with $g \wedge(t-g)^{+}>0$ and $t$ special. Then $2 t \wedge g$ is a nonzero component of $g$.

Proof. Consider a prime subgroup $R$ omitting $g \wedge(t-g)^{+}$. Then $R<R+g<$ $R+t$, hence $R \subseteq Q$, where $Q$ is the unique value of $t$, and this implies $Q+g \leqslant Q$ $+t<Q+2 t$. If $u=(g-(2 t \wedge g)) \wedge(2 t \wedge g)>0$, then for any prime $P$ omitting $u$ we would have $P<P+2 t<P+g$. Again, the fact that $t \notin P$ implies $P \subseteq Q$, which in turn implies $Q+2 t \leqslant Q+g$, a contradiction. Therefore $u=0$, proving $2 t \wedge g$ to be a component of $g$. $2 t \wedge g>0$ because $R+(2 t \wedge g)=R+g>R$, where $R$ has the meaning above.

Lemma 1.16. Suppose $a, b \in G^{+}$with $r=a \wedge(b-a)^{+}>0$. If $t$ is a nonzero component of $b$ such that $t^{\perp \perp} \subseteq r^{\perp \perp}$, then $t \wedge$ a is a nonzero component of $a$.

Proof. Let us show first that $u=(a-(a \wedge t)) \wedge(a \wedge t)=0$. If $u>0$, then since $u \leqslant t \in r^{\perp \perp}$ we may find a prime $P$ omitting $u \wedge r$, from which follows $P<P+t<P+a$. Now since $t$ is a component of $b$ we have $P+t=P+b<P$ $+a$, hence $(b-a)^{+} \in P$. But this contradicts the assumption that $P<P+r \leqslant P$ $+(b-a)^{+}$. We conclude $u=0$, meaning $t \wedge a$ is a component of $a .0 \neq t^{\perp \perp} \subseteq$ $r^{\perp \perp}$ implies $0 \neq t \wedge r \leqslant t \wedge a$.

Proposition 1.17. Suppose that $A$ and $B$ are l-subgroups of $G$ such that $B$ is convex and $A$ is saturated. Suppose further that $B$ satisfies either (a) or (b) below. Then $A$ lies above $B$ if and only if $A \cap B=0$.

(a) $b=\mathrm{V}(T \wedge b)$ for all $b \in B^{+}$, where $T=\left\{t \in B^{+}: t\right.$ special $\}$.

(b) $\mathscr{S}(B)$ is dense in $\mathscr{P}(B)$. That is, for each $0 \neq X \in \mathscr{P}(B)$ there is some $0 \neq Y \in \mathscr{S}(B)$ such that $Y \subseteq X$.

Proof. Suppose that $B$ satisfies (a) and that $r=a \wedge(b-a)^{+}>0$ for some $a \in A^{+}$and $b \in B^{+}$. Since $r=\bigvee\left\{(t \wedge(b-a))^{+} \wedge a: t \in T\right\}$, we may assume $b \in T$. By Lemma $1.15,2 b \wedge a$ is a nonzero component of $A$. $2 b \wedge a \in A$ by saturation and $2 b \wedge a \in B$ by convexity, so $A \cap B \neq 0$. Now suppose that $B$ satisfies (b) and that $r=a \wedge(b-a)^{+}>0$ for some $a \in A^{+}$and $b \in B^{+}$. Let $X=r^{\perp \perp}$ and find $0 \neq Y \in \mathscr{S}(B)$ with $Y \subseteq X$. Let $b=t+(b-t)$, where $t \in Y$ and $b-t \in Y^{\perp}$. By Lemma 1.16, $t \wedge a$ is a nonzero component of $a$, hence $t \wedge a \in A \cap B \neq 0$.

Let $\mathbf{S}$ designate the class of $l$-groups $G$ whose every positive element is a disjoint supremum of special elements, or equivalently, those $l$-groups $G$ having a plenary set of special subgroups. It is known (Theorem 3.18 of [3]) that $G$ is normal-valued and completely distributive if and only if $G$ is a large $l$-subgroup of a member of $\mathbf{S}$.

Corollary 1.18. Suppose that $A$ and $B$ are l-subgroups of $G$ such that $B$ is convex and that $A$ is saturated. Suppose further that either $B \in \mathbf{S}$ or $B$ is projectable. Then $A$ lies above $B$ if and only if $A \cap B=0$. 
We next show that $G$ is normal valued if and only if $G$ is an. $l$-subgroup of a member of $\mathbf{S}$. The proof modifies that of Proposition 3.17 of [3], and requires some auxiliary ideas.

Suppose $\Delta$ is a plenary set of regular subgroups of $G$ which is normal, that is, $-g+G_{\delta}+g \in \Delta$ whenever $G_{\delta} \in \Delta$ and $g \in G$. Then $\mathscr{C}(\Delta)=\left\{Y \subseteq G: Y \supseteq \cap \Delta_{0}\right.$ for finite $\left.\Delta_{0} \subseteq \Delta\right\}$ is the neighborhood filter of 0 of a topology (also designated $\mathscr{C}(\Delta)$ ) with respect to which all group and lattice operations are continuous [4]. The subbasic open sets have the form $G_{\delta}+g$ for $G_{\delta} \in \Delta$ and $g \in G$. We write $G \leqslant_{\Delta} H$ to mean that $H$ is an l-group having a normal plenary set $\Delta^{\prime}$ of regular subgroups such that $G$ is an $l$-subgroup of $H$ dense with respect to $\mathscr{C}\left(\Delta^{\prime}\right)$ and such that $\Delta^{\prime}$ and $\Delta$ are in one-one correspondence by intersection. The density condition may be reformulated as follows: any finite collection of cosets of elements in $\Delta^{\prime}$ which has nonempty intersection in $H$ must have nonempty intersection in $G$ also.

Proposition 1.19. Suppose $\Delta$ is a normal plenary set of regular subgroups of an l-group $G$. Then there is an l-group $G^{\Delta}$ which is unique up to l-isomorphism over $G$ with respect to the following property. For any l-group $H, G \leqslant_{\Delta} H$ if and only if there is an l-monomorphism $\theta: H \rightarrow G^{\Delta}$ over $G$.

Proof. Let $G^{\Delta}$ be the completion of $G$ with respect to the $l$-Cauchy structure $\mathscr{D}(\Delta)=\{\mathscr{F}: \mathscr{F}$ is a filter on $G$ such that $(\mathscr{F}-\mathscr{F}) \cap(-\mathscr{F}+\mathscr{F}) \supseteq \mathscr{C}(\Delta)\}$ induced by $\mathscr{C}(\Delta)[1]$. For each $G_{\delta} \in \Delta$ let $\operatorname{cl}\left(G_{\delta}\right)=\left\{[\mathscr{F}]: G_{\delta} \in \mathscr{F}\right\}$, a prime subgroup of $G^{\Delta}$, and let $\Delta^{\prime}=\left\{\operatorname{cl}\left(G_{\delta}\right): \delta \in \Delta\right\}$. It can readily be verified that $G \leqslant{ }_{\Delta} G^{\Delta}$. Now suppose $G \leqslant_{\Delta} H$ and consider $h \in H$. Let $\mathscr{F}$ be the trace of the neighborhood filter (with respect to $\mathscr{C}\left(\Delta^{\prime}\right)$ ) of $h$ on $G$, and let $\rightarrow$ designate convergence in $\mathscr{C}\left(\Delta^{\prime}\right)$. Then $\mathscr{F}-\mathscr{F} \rightarrow h-h=0$ and $-\mathscr{F}+\mathscr{F} \rightarrow-h+h=0$, so $\mathscr{F} \in \mathscr{D}(\Delta)$. Define $h \theta=$ [F]. One easily verifies that $\theta$ is an $l$-monomorphism.

Proposition 1.20. $G$ is normal valued if and only if $G^{\Delta} \in \mathbf{S}$, and in this case $\Delta^{\prime}$ is the minimal plenary set of special subgroups of $G^{\Delta}$.

Proof. Let $H$ be $G^{\Delta}$, and consider $0<h \in H$. Then $h=[\mathscr{H}]$ for some Cauchy filter $\mathscr{H}$ on $G$. Fix $\delta \in \Delta$ for which $H_{\delta}$ is a value of $h$ in $\Delta^{\prime}$. Let $\mathscr{F}$ be $\mathscr{M} \cup \mathscr{N}$, where $\mathscr{M}=\left\{G_{\gamma}+g_{\gamma} \in \mathscr{H}: G_{\gamma} \subseteq G_{\delta}\right\}$ and $\mathscr{N}=\left\{G_{\gamma}: G_{\gamma}\right.$ unrelated to $\left.G_{\delta}\right\}$. We claim that every finite subset of $\mathscr{F}$ has nonempty intersection. Given finite $\mathscr{N}_{1} \subseteq \mathscr{N}$ find $0<x_{\gamma} \in G_{\gamma} \backslash G_{\delta}$ for each $G_{\gamma} \in \mathscr{N}_{1}$ and let $x=\wedge x_{\gamma} \in\left(\cap G_{\gamma}\right) \backslash G_{\delta}$. Given finite $\mathscr{M}_{1} \subseteq \mathscr{M}$ choose $g \in \mathscr{M}_{1}$, possible since $\mathscr{M}_{1} \subseteq \mathscr{H}$. Because $G$ has normal values there is some positive integer $n$ such that $G_{\delta}+g<G_{\delta}+n x$. Therefore $n x \wedge g \in\left(\cap \mathscr{H}_{1}\right) \cap\left(\cap \mathscr{N}_{1}\right) \neq \varnothing$. This completes the proof of the claim that $\mathscr{F}$ generates a proper filter on $G$, one which is readily seen to be in $\mathscr{D}(\Delta)$. Let $[\mathscr{F}]=f \in H$. Now for $\gamma \in \Delta, G_{\gamma} \subseteq G_{\delta}$ implies the existence of some $g_{\gamma} \in G$ for which $G_{\gamma}+g_{\gamma} \in \mathscr{H} \cap \mathscr{F}$, hence $G_{\gamma} \in \mathscr{H}-\mathscr{F}$, while $G_{\gamma}$ unrelated to $G_{\delta}$ implies $G_{\gamma} \in \mathscr{F}$. Therefore $(\mathscr{H}-\mathscr{F}) \wedge \mathscr{F} \supseteq \mathscr{C}(\Delta)$, meaning that $(h-f) \wedge f=0$ so that $f$ is a component of $h$, and $h \in H_{\gamma}$ for all $\gamma \in \Delta$ such that $G_{\gamma}$ is unrelated to $G_{\delta}$, while $h-f \in H_{\gamma}$ for all $\gamma \in \Delta$ such that $G_{\gamma} \subseteq G_{\delta}$. Therefore $h$ is special with sole value $H_{\delta}$, which must therefore be closed. Clearly $h$ must be the supremum of its special components. 
To be sure, not all members of $\mathbf{S}$ have the form $G^{\Delta}$ for some l-group $G$. It is not difficult to show, for example, that $G^{\Delta}$ must be sup $\mathscr{Y}$ complete and therefore laterally complete. But $G^{\Delta}$ need not be Dedekind MacNeille complete; in particular, if $G$ is the $l$-group of rational numbers, then $G^{\Delta}=G$. Is it possible to characterize l-groups of the form $G^{\Delta}$ ?

In the next result, $A^{\sim}$ designates the convexification and $[A]$ designates $\cap\{C$ : $A \leqslant C \leqslant G$ and $C$ saturated $\}$ for an $l$-subgroup $A$ of $G$.

THEOREM 1.21. Suppose $A$ and $B$ are l-subgroups of the normal-valued l-group $G$. Then $A$ lies above $B$ if and only if $[A] \cap B \sim=0$ in $G^{\Delta}$. In particular, if $G \in \mathbf{S}$, then $a$ saturated subgroup $A$ lies above $B \in \mathscr{C}(G)$ if and only if $A \cap B=0$.

Proof. Suppose $G \in \mathbf{S}$ and that $\Delta$ is the minimal plenary set of special subgroups of $G$. Suppose that the saturated subgroup $A$ fails to lie above the convex $l$-subgroup $B$. By Proposition 1.4 there is some $a \in A^{+}, b \in B^{+}$, and $\delta \in \Delta$ such that $\delta \in \Delta(a) \cap \Delta(b)$. Because $G \in \mathbf{S}$ we may replace $a$ by its special component having value $\delta$, which also lies in $A$ because $A$ is saturated. Because $G^{\delta} / G_{\delta}$ is archimedean, $a \leqslant n b$ for some positive integer $n$. That is, $a \in A \cap B \neq \varnothing$.

2. Maximal below subgroups. In this section we present conditions sufficient for an $l$-group $B$ to be maximal below in $G$. Given another $l$-subgroup $A$ the notation $B \vee A^{\perp}$ refers to $\left\{g \in G:|g| \leqslant n(b \vee x)\right.$ for some $0<b \in B, 0<x \in A^{\perp}$, and positive integer $n\}$, the supremum of $B$ and $A^{\perp}$ in $\mathscr{C}(G)$.

Proposition 2.1. For l-subgroups $A$ and $B$ with $B$ convex, (a) implies (b), and (b) and (c) are equivalent. If $G=A^{\perp \perp} \boxplus A^{\perp}$ then (b) implies (a).

(a) $B \vee A^{\perp}$ is maximal below $A$ in $G$.

(b) $B \cap A^{\perp \perp}$ is maximal below $A$ in $A^{\perp \perp}$.

(c) $B \cap A^{\perp \perp}$ is maximal below $A \boxplus A^{\perp}$ in $G$.

Proof. Assuming (a) holds, $B \cap A^{\perp \perp}$ surely lies below $A$ in $A^{\perp \perp}$. If $B \cap A^{\perp \perp}$ $\subsetneq C \in \mathscr{C}\left(A^{\perp \perp}\right)$, then $C \vee A^{\perp} \supsetneq B \vee A^{\perp}$ by virtue of the distributivity of $\mathscr{C}(G)$; hence there are elements $a \in A^{+}, c \in C^{+}, 0<x \in A^{\perp}$, a prime subgroup $P$, and a positive integer $n$ such that $P<P+a<P+n(c \vee x)=P+n c \vee n x=P+n c$. $(n(c \vee x)=n(c+x)=n c+n x=n c \vee n x$ because $c \wedge x=0$, and $n x \in P$ because $a \wedge x=0$ and $a \notin P$.) This proves that (b) holds. If (b) holds and $B \cap A^{\perp \perp}$ $\subseteq C \in \mathscr{C}(G)$ such that $C$ lies below $A \boxplus A^{\perp}$, then $C \subseteq A^{\perp \perp}$ by the last assertion of Proposition 1.4, and so $C=B \cap A^{\perp \perp}$, meaning (c) holds, and (c) clearly implies (b). Finally, suppose that $G=A^{\perp \perp} \boxplus A^{\perp}$ and that (b) holds. Given $0<a \in A$, $0<b \in B$, and $0<x \in A^{\perp}$ we may write $b=b \nu a+b \mu a$, where $b \nu a \ll a$. Therefore

$$
a \wedge(n(b \vee x)) \leqslant a \wedge(n(b+x))=a \wedge(n b \nu a+n(b \mu a+x))=n b v a \ll a,
$$

proving that $B \vee A^{\perp}$ lies below $A$. If $B \vee A^{\perp} \subseteq C \in \mathscr{C}(G)$ such that $C$ lies below $A$, then

$$
C=C \cap G=\left(C \cap A^{\perp \perp}\right) \boxplus\left(C \cap A^{\perp}\right)=\left(B \cap A^{\perp \perp}\right) \boxplus A^{\perp}=B \vee A^{\perp},
$$

where the penultimate equality holds by (b). 
The following example shows that (b) does not generally imply (a) in Proposition 2.1. Let $\Gamma=\{0,1,2, \ldots\}$ be ordered by declaring $0>2 n+1$ for $n=1,2,3, \ldots$. Let $G=\{g \in V(\Gamma, \mathbf{Z}):\{n \in \Gamma:(n) g \neq k\}$ is finite for some $k \in \mathbf{Z}\}$, let $A$ be $\langle a\rangle$ where (0) $a=1$ and $(n) a=0$ for $n>0$, and let $B=\{g \in G:(2 n) g=0$ for $n=0$, $1,2, \ldots\}$. Then $B$ is maximal below $A$ in $A^{\perp \perp}=A$, yet $B \vee A^{\perp}=\{g \in G$ : $0 \notin\{n \in \Gamma:(n) g \neq 0\}$ finite $\}$ is properly contained in $B_{1}=\{g \in G:(0) g=0\}$, and $B_{1}$ is (maximal) below $A$ in $G$.

Corollary 2.2. Suppose $A$ and $B$ are l-subgroups of $G$. Then $B$ is maximal below $A$ in $A^{\perp \perp}$ if and only if $B$ is maximal below $A \boxplus A^{\perp}$.

We refer to $(A, B)$ as a maximal above and below pair if $A$ is an $l$-subgroup of $G$ maximal above $B$ and $B$ is an $l$-subgroup of $G$ maximal below $A$.

Proposition 2.3. Suppose $(A, B)$ is a maximal above and below pair in $G$. If $B$ is prime, then $A$ must be totally ordered. In a normal-valued l-group $G, B$ is prime if and only if $A$ is a totally ordered set of special elements. In particular, if a prime subgroup of a normal-valued l-group is maximal below, then it is an intersection of special subgroups. Therefore a regular subgroup of a normal-valued l-group is maximal below if and only if it is special.

Proof. If $a_{1}$ and $a_{2}$ are disjoint elements of $A$, then one must lie in $B$, hence one is 0 . That is, $A$ is totally ordered. If $G$ is normal valued, then $B=\bigcap\left\{G_{\delta}\right.$ : $\delta \in \Delta(A)\}$, hence $\Delta(A)$ must be totally ordered and all elements of $A$ must be special.

To see that the normal-valued hypothesis cannot be omitted from the preceding result take $G$ to be $\{g \in A(\mathbf{R}): g+p=p+g\}$, where $(r) p=r+1$ for all $r \in \mathbf{R}$. Let $A=\langle a\rangle$, where $(r) a=r+2$, and let $B$ be $\{g \in G:(0) g=0\}$.

COROLlaRY 2.4. A normal-valued l-group is finite-valued if and only if every regular subgroup is maximal below.

As in $\$ 1$, let $\mathbf{S}$ designate the class of all $l$-groups $G$ whose every positive element is a disjoint supremum of special elements.

COROllary 2.5. If $G$ is normal-valued, completely distributive, and if every closed convex l-subgroup of $G$ is maximal below, then $G \in \mathbf{S}$.

Proof. Let $\Delta$ be the minimal plenary set of closed regular subgroups of $G$. Since each $G_{\delta}$ is maximal below, it is also special.

Proposition 2.6. A subgroup $B$ of an abelian l-group $G$ is prime and maximal below if and only if $B$ is an intersection of a chain of special subgroups.

Proof. Suppose $B=\bigcap\left\{G_{\lambda}: \lambda \in \Lambda\right\}$ for some chain $\Lambda$ of special subgroups. For each $\lambda \in \Lambda$ choose special $a_{\lambda} \in G^{\lambda} \backslash G_{\lambda}$, and let $A$ be $\left\langle a_{\lambda}: \lambda \in \Lambda\right\rangle$. Then $B=\bigcap\left\{G_{\lambda}: \lambda \in \Delta(A)\right\}$ is maximal below $A$.

Corollary 2.7. An abelian l-group is finite-valued if and only if each prime subgroup is maximal below. 
CoROllary 2.8. If $0<a$ is special with value $G_{\gamma}$, then $G_{\gamma} \cap a^{\perp \perp}$ is maximal below $\langle a\rangle \boxplus a^{\perp}$ and also maximal below $\langle a\rangle$ in $a^{\perp \perp}$.

Proof. Take $A$ to be $\langle a\rangle$ and $B$ to be $G_{\gamma}$ in Proposition 2.1.

The convex $l$-subgroup $M$ of the following lemma has been well studied. A proof can be found in [11], for example, of the final assertion of the following lemma.

LEMMA 2.9. Suppose $P$ is a prime subgroup of $G$ and let $M=\{g \in G:|g| \wedge q=0$ for some $q \in G \backslash P\}$. Then $M \in \mathscr{C}(G)$, and any convex l-subgroup $C$ which contains $M$ must be related to $P$. Thus $M=\cap\{Q \in \mathscr{C}(G): Q \subseteq P$ and $Q$ prime $\}$.

Proof. If $0<g_{1}, g_{2} \in M$ with $g_{i} \wedge q_{i}=0$ for $q_{1}, q_{2} \in G \backslash P$, then $\left(g_{1}+g_{2}\right) \wedge$ $\left(q_{1} \wedge q_{2}\right)=0$, and $q_{1} \wedge q_{2} \in G \backslash P$ by the primeness of $P$. Thus $M \in \mathscr{C}(G)$. Now suppose $M \subseteq C \nsubseteq P$ for some $C \in \mathscr{C}(G)$; say $c \in C^{+} \backslash P$. Consider $p \in P^{+}$. Then $c \wedge p \in P \cap C$, hence $c^{\prime}=c(c \wedge p)^{-1} \notin P$. Because $c^{\prime} \wedge p^{\prime}=0$, where $p^{\prime}=$ $p(c \wedge p)^{-1}$, we conclude that $p^{\prime} \in M \subseteq C$, whence $p \in C$. That is, $P \subseteq C$. Finally, let $\mathscr{M}=\{Q \in \mathscr{C}(G): Q \subseteq P, Q$ prime $\}$. Given $p \in P \backslash M$ there is some prime subgroup $Q$ such that $M \subseteq Q$ and $p \notin Q$, and the preceding argument shows $Q \in \mathscr{M}$. Therefore $\cap \mathscr{M} \subseteq M$. On the other hand, for any $Q \in \mathscr{M}, g \in G$, and $q \in G \backslash P$ such that $g \wedge q=0$ we must have $g \in Q$ by primeness. That is, $M \subseteq \cap \mathscr{M}$.

Proposition 2.10. If $G$ is abelian and $0<a \in G$ is special with value $G_{\gamma}$, then each $N \in \mathscr{C}(G)$ between $G_{\gamma} \cap a^{\perp \perp}$ and $a^{\perp \perp}$ is maximal below.

Proof. If $N=a^{\perp \perp}$, then $N$ is maximal below $a^{\perp}$ by Proposition 1.4, and if $N=G_{\gamma} \cap a^{\perp \perp}$, then $N$ is maximal below $\langle a\rangle \boxplus a^{\perp \perp}$ by Corollary 2.8, so assume $G_{\gamma} \cap a^{\perp \perp}<N<a^{\perp \perp}$. Observe that any $h \in a^{\perp \perp} \backslash G_{\gamma}$ must be special. (If $h$ had a value $G_{\delta}$ unrelated to $G_{\gamma}$, then by Lemma 2.9 there would be disjoint elements $x \in G_{\delta} \backslash G_{\gamma}$ and $y \in G_{\gamma} \backslash G_{\delta}$, and by replacing $x$ by some multiple we could assume $x>g$. Then $y \in g^{\perp} \backslash h^{\perp}$, contrary to assumption.) Let $\Gamma=\left\{G_{\gamma}: G_{\gamma}\right.$ is the value of some $\left.0<h \in a^{\perp \perp} \backslash N\right\}$ and for each choose $h_{\gamma} \in a^{\perp \perp} \backslash N$ with value $G_{\gamma}$. Let $A=\left\langle h_{\gamma}: \gamma \in \Gamma\right\rangle$. Since $N$ is maximal below $A$ in $a^{\perp \perp}$ by construction, $N$ is maximal below $A \boxplus a^{\perp}$ by Proposition 2.1.

Proposition 2.11. Suppose $\left\{G_{\lambda}: \lambda \in \Lambda\right\}$ is a set of special subgroups. If $G$ is abelian, or if each element of $\Lambda$ exceeds an element minimal in $\Lambda$, then $B=\bigcap\left\{G_{\gamma}\right.$ : $\lambda \in \Lambda\}$ is maximal below.

Proof. For each $\lambda \in \Lambda$ choose $a_{\lambda}$ to be a positive special element in $G^{\lambda} \backslash G_{\lambda}$. If $G$ is abelian, then the subgroup $A$ generated by the $a_{\lambda}$ 's is the direct sum of the cyclic subgroups $\left\langle a_{\lambda}\right\rangle$, hence $\Gamma(A)=\Lambda$, where $\Gamma$ here represents the plenary set of all regular subgroups of $G$. Therefore $B$ is maximal below $A$ by Theorem 1.6. If each element of $\Lambda$ exceeds an element in $\Lambda_{0}=\{\lambda \in \Lambda: \lambda$ is minimal in $\Lambda\}$, then let $A=\left\langle a_{\lambda}: \lambda \in \Lambda_{0}\right\rangle$. Observe that since $a_{\lambda} \wedge a_{\delta}=0$ for $\lambda \neq \delta$ in $\Lambda_{0}, A$ is the cardinal sum of the cyclic subgroups $\left\langle a_{\lambda}\right\rangle$ for $\lambda \in \Lambda_{0}$. If $0<a \in A$ and $0<b \in$ $\cap\left\{G_{\lambda}: \lambda \in \Lambda_{0}\right\}$ satisfy $P<P+a<P+b$, then the value of $a$ containing $P$ must lie in $\Lambda_{0}$ and therefore contains $b$, a contradiction. This shows that $\cap G_{\lambda}$ lies below 
$A$. If $G_{\lambda} \subset B \in \mathscr{C}(G)$, then there is some $\lambda \in \Lambda_{0}$ such that $B \nsubseteq G_{\lambda}$. Because $G_{\lambda}$ is normal in $G^{\lambda}$ (Corollary 4.3.5 and Proposition 4.4.4 of [6]), $G_{\lambda}+a_{\lambda}<G_{\lambda}+b$ for some $b \in B$, hence $a_{\lambda} \in B$, a contradiction.

COROllaRy 2.12. If $\Gamma(G)$ is finite, then every convex l-subgroup is maximal below.

LemMa 2.13. Suppose B is a convex l-subgroup of an abelian l-group $G$. If there is a plenary set $\Delta$ of regular primes such that for each $\delta \in \Delta \backslash \Delta(B)$ there is some $0<g(\delta) \in G$ with the property that $\{\gamma \in \Delta(g(\delta)): \gamma$ is related to an element of $\Delta(B)\} \subseteq\{\delta\} \subseteq \Delta(g(\delta))$, then $B^{\prime}=\{g \in G: \Delta(g) \subseteq \Delta(B)\}$ is maximal below.

Proof. An element of the group generated by $\{g(\delta): \delta \in \Delta \backslash \Delta(B)\}$ has the form $x=\sum_{i=1}^{k} n_{i} g\left(\delta_{i}\right)$, where $\delta_{i} \in \Delta \backslash \Delta(B)$ and $n_{i} \in \mathbf{Z}$. Suppose $x$ has value $\gamma$ related to an element $\lambda \in \Delta(B)$. By relabelling, assume $g\left(\delta_{i}\right) \notin G_{\gamma}$ for $1 \leqslant i \leqslant m$ and $g\left(\delta_{i}\right) \in G_{\gamma}$ for $m+1 \leqslant i \leqslant k$, so that $G_{\gamma}+x=G_{\gamma}+\sum_{i=1}^{m} n_{i} g\left(\delta_{i}\right)$. For each $i$, $1 \leqslant i \leqslant m, g\left(\delta_{i}\right)$ has a value $\rho_{i}$ containing $\gamma$, and since $\rho_{i}$ is also related to $\lambda$, $\rho_{i}=\delta_{i}$ in each case. Therefore $\gamma$ is the greatest element in the chain $\left\{\delta_{i}: 1 \leqslant i \leqslant m\right\}$, and in particular $\gamma \in \Delta \backslash \Delta(B)$. Since $\Delta(A)=\Delta \backslash \Delta(B)$ it follows that $B^{\prime}$ is maximal below $A$.

Proposition 2.14. If $g$ is a finite-valued element of an abelian l-group $G$, then $G(g)$ is maximal below.

Proof. Write $g$ as $g_{1}+g_{2}+\cdots+g_{n}$, where the $g_{i}$ 's are disjoint and special with respective values $\gamma_{1}, \gamma_{2}, \ldots, \gamma_{n}$. Given $\lambda \in \Gamma \backslash \Gamma(g)$ let $I=\left\{i: \lambda \ngtr \gamma_{i}\right\}$, and for each $i \in I$ choose $0<x_{i} \in\left(G^{\lambda} \backslash G_{\lambda}\right) \cap G_{\gamma_{i}}$. Let $g(\lambda)=\wedge\left\{\left(x_{i}-\left|g_{i}\right|\right)^{+}: i \in I\right\}$ if $I \neq \varnothing$, otherwise let $g(\lambda)$ be any positive element of $G^{\lambda} \backslash G_{\lambda}$. Now take $B=G(g)$ in Lemma 2.13, and note that $B^{\prime}=B$.

The descending chain condition (every descending chain is finite) is abbreviated DCC.

Proposition 2.15. Suppose $G \in \mathbf{S}$, and suppose $G$ is either abelian or that the minimal plenary set $\Delta$ satisfies the DCC. Then every closed convex l-subgroup is maximal below.

Proof. For $B \in \mathscr{K}(G)$ we claim $B=\bigcap\left\{G_{\delta}: \delta \in \Delta, B \subseteq G_{\delta}\right\}$. For if $0<x \notin B$ then $x=\mathrm{V} T$ for some disjoint set $T$ of special elements. Since $B$ is closed it cannot contain $T$, say $0<t \in T \backslash B$ with value $\delta \in \Delta$. Then $B \subseteq G_{\delta}$, since otherwise $t \in B$, and this proves the claim. To prove the proposition take $\Gamma=\{\delta \in \Delta$ : $\left.B \subseteq G_{\delta}\right\}$ in Proposition 2.11.

COROLlARY 2.16. If $G$ is finite-valued and its plenary set of regular subgroups enjoys the $D C C$, then every convex l-subgroup of $G$ is maximal below.

The example following Theorem 2.19 shows that the DCC cannot be omitted from the hypotheses of the previous corollary. In the following proposition, $\bar{B}=$ $\bigcap\{C \in \mathscr{K}(G): B \subseteq C\}$ denotes the closure of $B$. It is known that $\bar{B}=\{g \in G$ : $\left.|g|=\bigvee\left\{b \in B^{+}: b \leqslant|g|\right\}\right\}$. 
LEMMA 2.17. Suppose $G$ is normal-valued and completely distributive with minimal plenary set $\Delta$. Then $\bar{B}=\bigcap\left\{G_{\delta}: B \subseteq G_{\delta}\right.$ and $\left.\delta \in \Delta\right\}=\{g \in G: \Delta(g) \subseteq \Delta(B)\}$.

Proof. The first equality follows from the fact that any closed convex $l$-subgroup of a normal-valued completely distributive l-group is an intersection of closed prime subgroups [14]. The second equality can be routinely verified.

Proposition 2.18. Suppose $G$ is an abelian l-group in $\mathbf{S}$. If $A$ is a subgroup maximal above $B \in \mathscr{C}(G)$, then $\bar{B}$ is maximal below $A$.

Proof. $\bar{B}$ lies below $A$ by Theorem 1.6. Suppose $\bar{B} \subsetneq H \in \mathscr{C}(G)$ and that $H$ lies below $A$. Fix $0<h \in H \backslash \bar{B}$, and write $h=\bigvee T$ for some disjoint set $T$ of special elements. Now $T \nsubseteq \bar{B}$, for otherwise the closure of $\bar{B}$ would imply $h \in \bar{B}$. Fix $t \in T \backslash \bar{B}$ with value $\gamma$. We claim that the subgroup $A^{\prime}$ generated by $A \cup\{t\}$ lies above $B$. Consider a value $\delta$ of a typical element $a+n t \in A^{\prime}$, where $a \in A$ and $n$ is an integer. If $t \in G_{\delta}$, then $\delta$ is a value of $a$ and so $G_{\delta} \supseteq B$. If $t \notin G_{\delta}$, then $\delta \leqslant \gamma$, and by Proposition $1.2 \gamma<\alpha$ for some value $\alpha$ of $a$. Therefore $\delta=\alpha$, and again $G_{\delta} \supseteq B$. This proves the claim. But then the maximality of $A$ forces $t \in A$, contradicting the assumption that $H$ lies below $A$. We can only conclude that $\bar{B}=H$.

The following theorem sums up the preceding results.

THEOREM 2.19. Suppose $G$ is normal-valued and completely distributive with minimal plenary set $\Delta$. If $G$ is either abelian or $\Delta$ has the DCC, then each closed convex l-subgroup of $G$ is maximal below if and only if $G$ is in $\mathbf{S}$. If $G$ is abelian and in $\mathbf{S}$ and if $A$ is a subgroup maximal above $B \in \mathscr{C}(G)$, then $\bar{B}=\{g \in G: \Delta(g) \subseteq \Delta(B)\}=$ $\bigcap\left\{G_{\delta}: \delta \in \Delta\right.$ and $\left.B \subseteq G_{\delta}\right\}$ is maximal below $A$.

We close this section with an example which shows that the hypothesis that $G$ be either abelian or have a root system with the DCC cannot be dropped from the last several results. This example also shows that a normal convex $l$-subgroup of a totally ordered group need not be maximal below. Let $G$ be the free group on a countably infinite set of generators and let $B=[G, G]$ be its commutator subgroup. Then $G / B$ is group isomorphic to $\sum_{i=1}^{\infty} \mathbf{Z}_{i}$ and $G$ can be totally ordered so that $B$ is convex and $G / B$ is $o$-isomorphic to $\Sigma\left(\omega^{*}, \mathbf{Z}\right)$, where $\omega^{*}$ is the set of integers under reverse order. The point here is that $G / B$ has no smallest nontrivial convex $l$-subgroup.

Now suppose $B$ is maximal below $A$ and pick $u, v \in A$. Then $u+v-u-v \in A$ $\cap B=0$, meaning $A$ is abelian. Since $A$ is also free, $A=\langle a\rangle$. If $G_{\alpha}$ is the value of $a$ in $G$ we get $B \subsetneq G_{\alpha}$ below $A$. Thus $B$ is not maximal below. Observe that this example shows that the set of maximal below subgroups of $G$ is not closed under intersection.

The construction above can be modified to produce a totally ordered group $H$ whose every convex $l$-subgroup is normal, but whose only maximal below subgroups are the regular ones. Let $H$ be the free group on countably many generators as before, and let $H=H^{(0)} \geqslant H^{(1)} \geqslant \cdots$ be its descending central series. Then $H$ can be totally ordered so that each $H^{(n)}$ is convex and so that each quotient $H^{(n)} / H^{(n+1)}$ 
is order isomorphic to $\Sigma\left(\omega^{*}, \mathbf{Z}\right)$. An argument similar to the one above shows that $H$ has the stated properties.

3. The construction of the splitting extension. Suppose $l$-groups $A$ and $B$ are given along with a group homomorphism $\sigma$ from $A$ into the group of $l$-automorphisms of $B$. Can the resulting group splitting extension of $B$ by $A$, denoted $A \times B$, be endowed with a lattice order extending those on $A$ and $B$ and with respect to which $A$ lies above $B$ ? We prove that the answer to this question is affirmative, and that every such order is uniquely determined by a lattice homomorphism $\pi: \mathscr{C} p(A) \rightarrow$ $\mathscr{S}(B)$ such that $\pi(0)=0$. The construction of $G=A \times B$ from $A, B, \sigma$, and $\pi$ is shown to be sufficiently general to encompass cardinal product, lex extension, and $l$-permutation wreath product.

Lemma 3.1. Suppose a lies above $b$ in G. Then $(a+b)^{+}=a^{+}+a^{+} \wedge b^{+}-a^{+} \wedge b^{-}$ $+(b \mu a)^{+}$and $(a+b)^{-}=a^{-} \wedge b^{-}-a^{-} \wedge b^{+}+a^{-}+(b \mu a)^{-}$. Thus $a+b \geqslant 0$ if and only if $a \geqslant 0$ and $b \mu a \geqslant 0$.

Proof. By substituting the expression for $b \nu a+b \mu a$ in Proposition 1.1 for $b$ in $a+b$ and grouping, one obtains $a+b=c-d+b \mu a$, where $c=a^{+}+a^{+} \wedge b^{+}-$ $a^{+} \wedge b^{-}$and $d=a^{-} \wedge b^{-}-a^{-} \wedge b^{+}+a^{+}$. Now $a^{+}=0$ implies $c=0$ and $a^{+}>0$ implies $c=a^{+}+a^{+} \wedge b^{+}-a^{+} \wedge b^{-} \geqslant a^{+}-a^{+} \wedge b^{-}>0$ because $b^{-}$lies below $a^{+}$. Likewise $a^{-}=0$ implies $d=0$ and $a^{-}>0$ implies $d>0$. Since $c$ and $d$ are disjoint, we get $(a+b)^{+}=c+(b \mu a)^{+}$and $(a+b)^{-}=d+(b \mu a)^{-}$. Clearly $(a+b)^{-}=0$ if and only if $a^{-}=(b \mu a)^{-}=0$.

TheOREM 3.2. Suppose $A$ and $B$ are l-subgroups of $G$ such that $A$ is above $B$ and let $H$ be the subgroup generated by $A$ and $B$. If $B$ is normal in $H$, then $H$ is a splitting extension of $B$ by $A$, and the following are equivalent.

(a) $H$ is an l-subgroup of $G$.

(b) $a \wedge b \in B$ for all $a \in A^{+}$and $b \in B^{+}$.

(c) $B=B \cap H(a) \boxplus B \cap a^{\perp}$ for all $a \in A$.

Furthermore, $B$ is maximal below $A$ and $A$ is maximal above $B$ in $H$. In particular, if $G$ is normal-valued and if $B$ is maximal below $A$ in $G$, then $B$ is normal in $H$ and the conditions above hold.

Proof. Suppose $B$ is normal in $H$. Then $H$ is a splitting extension of $B$ by $A$ because $B \cap A=0$. Now assume (a) and consider $a \in A^{+}$and $b \in B^{+}$. Then $a \wedge b=a_{1}+b_{1}$ for some $a_{1} \in A$ and $b_{1} \in B$. We claim that $a_{1}=0$, for if not there is some prime $P$ omitting $a_{1}$. $P$ also omits $a_{1}+b_{1}$, for otherwise we would have $P<P+a_{1}=P-b_{1}<P-2 b_{1}$ or perhaps $P<P-a$, meaning $Q<Q-$ $a_{1}=Q+b_{1}<Q+2 b_{1}$ where $Q=-a_{1}+P+a_{1}$, in either case a violation of Proposition 1.1(c). So $P<P+a_{1}+b_{1}=P+a \wedge b=P+b$, hence $P<P+a_{1}$ $=P+\left(b-b_{1}\right)<P+2\left(b-b_{1}\right)$, again contradicting Proposition 1.1(c). This proves that $a \wedge b=b_{1} \in B$. If (b) holds then $b \nu a \in B$ by Proposition 1.1 and so (c) holds. If $a \in A^{+}$and $b \in B^{+}$and (c) holds, then $b=b_{1}+b_{2}$ where $b_{1} \in B \cap H_{(a)}$ and $b_{2} \in B \cap a^{\perp}$, but by Proposition 1.1, $b_{1}=b \nu a=b \wedge a \in B$, hence (b) holds. 
And (b) implies (a) by Lemma 3.1. Finally, observe that $A$ is maximal above $B$ in $H$ in the very strong sense that any subgroup of $H$ properly containing $A$ has nontrivial intersection with $B$, and $B$ is likewise maximal below $A$ in $H$.

If $B$ is maximal below $A$ in the normal-valued $l$-group $G$ and if $a \in A^{+}$and $b \in B^{+}$, then $b \wedge\left(a+a_{1}-a\right) \ll a+a_{1}-a$ implies $(-a+b+a) \wedge a_{1} \ll a_{1}$. This shows that $B$ is normal in $H$, and the convexity of $B$ in $G$ implies that conditions (a), (b), and (c) hold in this case.

Suppose $A, B$, and $H$ satisfy the conditions of Theorem 3.2. With each $a \in A$ associate the $l$-automorphism $\sigma(a)$ of $B$ defined by $b \sigma(a)=-a+b+a$. Then the addition in $H=A+B$ is given by

$$
\left(a_{1}+b_{1}\right)+\left(a_{2}+b_{2}\right)=\left(a_{1}+a_{2}\right)+\left(b_{1} \sigma\left(a_{2}\right)+b_{2}\right) .
$$

With each $a \in A$ also associate $[a]=B \cap H(a)=\{b \in B:|b| \ll|a|\} \in \mathscr{S}(B)$. Denote the complementary summand $[a]^{\prime}=B \cap a^{\perp}$. Let $\pi: \mathscr{C} p(A) \rightarrow \mathscr{S}(B)$ be defined by $\pi(A(a))=[a]$. Note that $\pi$ is well defined since $A\left(a_{1}\right)=A\left(a_{2}\right)$ implies $H\left(a_{1}\right)=H\left(a_{2}\right)$. Associated with the summand $[a]$ are the projection $l$-epimorphisms $\nu a: B \rightarrow[a]$ and $\mu a: B \rightarrow[a]^{\prime}$ satisfying $b=b \nu a+b \mu a$ for all $b \in B$. The following proposition, whose proof is straightforward, sets down the relevant attributes of $\sigma$ and $\pi$.

Proposition 3.3. Suppose $A$ and $B$ are l-subgroups of $G$ such that $B$ lies below $A, B$ is normal in the group $H$ generated by $A \cup B$, and $H$ is an l-subgroup of $G$. Then $\sigma$ is $a$ group homomorphism from $A$ into the group of l-automorphisms of $B, \pi$ is a lattice homomorphism from $\mathscr{C} p(A)$ into $\mathscr{S}(B)$, and the following properties hold.

(a) $\pi(0)=0$.

(b) $\sigma(a)$ is the identity on $[a]^{\prime}$ for all $a \in A$.

(c) $\left[a_{1}\right] \sigma\left(a_{2}\right)=\left[-a_{2}+a_{1}+a_{2}\right]$ for all $a_{1}, a_{2} \in A$.

The following theorem, which is a converse of Proposition 3.3, is one of the central results of this paper. It asserts that the properties of $\sigma$ and $\pi$ pointed out above are the important ones, for they are sufficient to construct $H$ from $A, B, \sigma$, and $\pi$.

THEOREM 3.4. Suppose $A$ and $B$ are l-groups, $\sigma$ is a group homomorphism from $A$ into the group of l-automorphisms of $B$, and $\pi$ is a lattice homomorphism from $\mathscr{C} p(A)$ into $\mathscr{S}(B)$, where $\pi[A(a)]$ is denoted by [a]. Suppose further that properties (a), (b), and (c) of Proposition 3.3 hold. Let $G=A \times B$, where $\left(a_{1}, b_{1}\right)+\left(a_{2}, b_{2}\right)=\left(a_{1}+\right.$ $\left.a_{2}, b_{1} \sigma\left(a_{2}\right)+b_{2}\right)$, and define $(a, b) \geqslant 0$ if and only if $a \geqslant 0$ and $b \mu a \geqslant 0$. Then $G$ is an l-group in which $A$ and $B$ are embedded as l-subgroups such that $A$ is maximal above $B$ and $B$ is maximal below $A$. Moreover bo $(a)=-a+b+a$ and $\pi[A(a)]=[a]=$ $G(a) \cap B$ for all $a \in A$ and $b \in B$.

References to $A$ and $B$ as subgroups of $G$ in Theorem 3.4 presume the usual identification of each $a \in A$ with $(a, 0) \in G$ and each $b \in B$ with $(0, b) \in G$. We shall prove this theorem by a sequence of lemmas, each of which assumes the notation and terminology of Theorem 3.4. The first lemma in the sequence proves 
the antisymmetry of the order; it makes use of the fact that $[0]^{\prime}=B$ because $[0]=\pi(0)=0$, hence $b \nu 0=0$ and $b \mu 0=b$ for all $b \in B$. Note also that $-(a, b)=$ $(-a,-b \sigma(-a))$ in $G$.

LEMMA 3.5. If $(a, b) \geqslant 0$ and $-(a, b) \geqslant 0$, then $a=0$ and $b=0$.

Proof. $(a, b) \geqslant 0$ implies $a \geqslant 0$, and $-(a, b)=(-a,-b \sigma(-a)) \geqslant 0$ implies $a \leqslant 0$, so $a=0$. And $(a, b) \geqslant 0$ also implies $b \mu a=b \mu 0=b=0$.

The next lemma proves the closure of the positive cone under addition. It makes use of the fact that for $a_{1}, a_{2} \in A$ and $b \in B, b \nu a_{1} \sigma\left(a_{2}\right)$ is a component of $b \sigma\left(a_{2}\right)$ because $b \nu a_{1}$ is a component of $b$. Since $\left[a_{1}\right] \sigma\left(a_{2}\right)=\left[-a_{2}+a_{1}+a_{2}\right]$, we see $b \nu a_{1} \sigma\left(a_{2}\right)=b \sigma\left(a_{2}\right) \nu\left(-a_{2}+a_{1}+a_{2}\right)$ and $b \mu a_{1} \sigma\left(a_{2}\right)=b \sigma\left(a_{2}\right) \mu\left(-a_{2}+a_{1}+a_{2}\right)$. In addition if $A\left(a_{1}\right) \subseteq A\left(a_{2}\right)$, then $\left[a_{1}\right]=\pi\left(A\left(a_{1}\right)\right) \subseteq \pi\left(A\left(a_{2}\right)\right)=\left[a_{2}\right]$. So under these circumstances $b \nu a_{2} \nu a_{1}=b \nu a_{1} \nu a_{2}=b \nu a_{1}$ and $b \mu a_{2} \mu a_{1}=b \mu a_{1} \mu a_{2}=b \mu a_{2}$, hence $b \nu a_{2} \geqslant 0$ implies $b \nu a_{1}=0$ and $h \mu a_{1}=0$ implies $b \mu a_{2}=0$.

Lemma 3.6. If $\left(a_{1}, b_{1}\right),\left(a_{2}, b_{2}\right) \geqslant 0$, then $\left(a_{1}, b_{1}\right)+\left(a_{2}, b_{2}\right) \geqslant 0$.

Proof. Clearly $a_{1}+a_{2} \geqslant 0$ because $a_{1}, a_{2} \geqslant 0$, and

$$
\begin{aligned}
\left(b_{1} \sigma\left(a_{2}\right)+b_{2}\right) \mu\left(a_{1}+a_{2}\right) & =b_{1} \sigma\left(a_{2}\right) \mu\left(a_{1}+a_{2}\right)+b_{2} \mu\left(a_{1}+a_{2}\right) \\
& =b_{1} \mu\left(a_{2}+a_{1}\right) \sigma\left(a_{2}\right)+b_{2} \mu\left(a_{1}+a_{2}\right) \\
& \geqslant 0
\end{aligned}
$$

because $b_{1} \mu a_{1}, b_{2} \mu a_{2} \geqslant 0$ imply $b_{1} \mu\left(a_{1}+a_{2}\right)$ and $b_{2} \mu\left(a_{1}+a_{2}\right) \geqslant 0$.

The next lemma establishes the normality of the positive cone.

LEMMA 3.7. If $\left(a_{2}, b_{2}\right) \geqslant 0$, then $-\left(a_{1}, b_{1}\right)+\left(a_{2}, b_{2}\right)+\left(a_{1}, b_{1}\right) \geqslant 0$ for any $\left(a_{1}, b_{1}\right) \in G$.

ProOF.

$$
-\left(a_{1}, b_{1}\right)+\left(a_{2}, b_{2}\right)+\left(a_{1}, b_{1}\right)=\left(a,-b_{1} \sigma(a)+b_{2} \sigma\left(a_{1}\right)+b_{1}\right),
$$

where $a=-a_{1}+a_{2}+a_{1} \geqslant 0$, and

$$
\begin{aligned}
-b_{1} \sigma(a) \mu a+b_{2} \sigma\left(a_{1}\right) \mu a+b_{1} \mu a & =-b_{1} \mu a \sigma(a)+b_{2} \mu a_{2}+b_{1} \mu a \\
& =-b_{1} \mu a+b_{2} \mu a_{2}+b_{1} \mu a \geqslant 0,
\end{aligned}
$$

where the last equality holds because $\sigma(a)$ is the identity on $[a]^{\prime}$.

Thus far we have shown $G$ to be a partially ordered group. We shall show outright that $G$ is a lattice in Lemma 3.9, which is made easier by the following result.

LEMMA 3.8. $\left(a_{1}, b_{1}\right) \geqslant\left(a_{2}, b_{2}\right)$ if and only if $a_{1} \geqslant a_{2}$ and $b_{1} \mu\left(-a_{2}+a_{1}\right) \geqslant$ $b_{2} \mu\left(-a_{2}+a_{1}\right)$.

ProOF.

$$
\left(a_{1}, b_{1}\right)-\left(a_{2}, b_{2}\right)=\left(a_{1}-a_{2},\left(b_{1}-b_{2}\right) \sigma\left(-a_{2}\right)\right) \geqslant 0
$$

if and only if $a_{1}-a_{2} \geqslant 0$ and

$$
\left(b_{1}-b_{2}\right) \sigma\left(-a_{2}\right) \mu\left(a_{1}-a_{2}\right)=\left(b_{1}-b_{2}\right) \mu\left(-a_{2}+a_{1}\right) \sigma\left(-a_{2}\right) \geqslant 0 .
$$


But this is equivalent to $a_{1} \geqslant a_{2}$ and $\left(b_{1}-b_{2}\right) \mu\left(-a_{2}+a_{1}\right) \geqslant 0$, which is the condition above.

LEMMA 3.9.

$$
\begin{aligned}
& \left(a_{1}, b_{1}\right) \vee\left(a_{2}, b_{2}\right) \\
& \quad=\left(a_{1} \vee a_{2}, b_{1} \nu\left(-a_{2}+a_{1}\right)^{+}+b_{2} \nu\left(-a_{1}+a_{2}\right)^{+}+\left(b_{1} \vee b_{2}\right) \mu\left(-a_{2}+a_{1}\right)\right), \\
& \left(a_{1}, b_{1}\right) \wedge\left(a_{2}, b_{2}\right) \\
& \quad=\left(a_{1} \wedge a_{2}, b_{2} \nu\left(-a_{2}+a_{1}\right)^{+}+b_{1} \nu\left(-a_{1}+a_{2}\right)^{+}+\left(b_{1} \wedge b_{2}\right) \mu\left(-a_{2}+a_{1}\right)\right) .
\end{aligned}
$$

Proof. Abbreviate by $\left(a_{1} \vee a_{2}, b\right)$ the expression for $\left(a_{1}, b_{1}\right) \vee\left(a_{2}, b_{2}\right)$ above. We claim $\left(a_{1} \vee a_{2}, b\right) \geqslant\left(a_{1}, b_{1}\right)$ for $a_{1} \vee a_{2} \geqslant a_{1}$ and

$$
\begin{aligned}
b \mu\left(-a_{1}+a_{1} \vee a_{2}\right) & =b \mu\left(-a_{1}+a_{2}\right)^{+}=b_{1} \nu\left(-a_{2}+a_{1}\right)^{+}+\left(b_{1} \vee b_{2}\right) \mu\left(-a_{2}+a_{1}\right) \\
& \geqslant b_{1} \nu\left(-a_{2}+a_{1}\right)^{+}+b_{1} \mu\left(-a_{2}+a_{1}\right) .
\end{aligned}
$$

Now $A\left(\left(-a_{2}+a_{1}\right)^{+}\right) \vee A\left(\left(-a_{1}+a_{2}\right)^{+}\right)=A\left(-a_{1}+a_{2}\right)$ and $A\left(\left(-a_{2}+a_{1}\right)^{+}\right) \cap$ $A\left(\left(-a_{1}+a_{2}\right)^{+}\right)=0$, and since $\pi$ is a lattice homomorphism we have $\left[\left(-a_{2}+a_{1}\right)^{+}\right]$ $\boxplus\left[\left(-a_{1}+a_{2}\right)^{+}\right]=\left[-a_{1}+a_{2}\right]$ and $\left[\left(-a_{2}+a_{1}\right)^{+}\right] \cap\left[\left(-a_{1}+a_{2}\right)^{+}\right]=0$, from which follows $\left[\left(-a_{2}+a_{1}\right)^{+}\right] \boxplus\left[-a_{1}+a_{2}\right]^{\prime}=\left[\left(-a_{1}+a_{2}\right)^{+}\right]^{\prime}$. Therefore $b_{1} \nu\left(-a_{2}+a_{1}\right)^{+}+$ $b_{1} \mu\left(-a_{2}+a_{1}\right)=b_{1} \mu\left(-a_{1}+a_{2}\right)^{+}$, which completes the verification of the claim. A similar argument shows $\left(a_{1} \vee a_{2}, b\right) \geqslant\left(a_{2}, b_{2}\right)$.

Suppose $\left(a_{3}, b_{3}\right) \geqslant\left(a_{1}, b_{1}\right), \quad\left(a_{2}, b_{2}\right)$. Clearly $a_{3} \geqslant a_{1} \vee a_{2}$, and we know $b_{3} \mu\left(-a_{1}+a_{3}\right) \geqslant b_{1} \mu\left(-a_{1}+a_{3}\right)$ and $b_{3} \mu\left(-a_{2}+a_{3}\right) \geqslant b_{3} \mu\left(-a_{2}+a_{3}\right)$. We must show $b_{3} \mu a \geqslant b \mu a$, where $a=-a_{1} \vee a_{2}+a_{3}=\left(-a_{1}+a_{3}\right) \wedge\left(-a_{2}+a_{3}\right)$. Since $\left[\left(-a_{2}+a_{1}\right)^{+}\right] \boxplus\left[\left(-a_{1}+a_{2}\right)^{+}\right] \boxplus\left[-a_{2}+a_{1}\right]^{\prime}=B$, this amounts to showing that each term of $b_{3} \mu a=b_{3} \nu\left(-a_{2}+a_{1}\right)^{+} \mu a+b_{3} \nu\left(-a_{1}+a_{2}\right)^{+} \mu a+b_{3} \mu\left(-a_{2}+a_{1}\right) \mu a$ exceeds $(\geqslant 0)$ the corresponding term of $b \mu a$. We begin by showing

$$
b_{3} \nu\left(-a_{2}+a_{1}\right)^{+} \mu a \geqslant b_{1} \nu\left(-a_{2}+a_{1}\right)^{+} \mu a \text {. }
$$

Now $0 \leqslant\left(-a_{2}+a_{1}\right)^{+}=-a_{2}+\left(a_{1} \vee a_{2}\right) \leqslant-a_{2}+a_{3}$, so $\left[\left(-a_{2}+a_{1}\right)^{+}\right] \subseteq$ $\left[-a_{2}+a_{3}\right]$ or $\left[\left(-a_{2}+a_{2}\right)^{+}\right] \cap\left[-a_{2}+a_{3}\right]^{\prime}=0$, which together with $\left[-a_{1}+a_{3}\right]^{\prime} \vee$ $\left[-a_{2}+a_{3}\right]^{\prime}=[a]^{\prime}$ yields $\left[\left(-a_{2}+a_{1}\right)^{+}\right] \cap[a]^{\prime} \subseteq\left[-a_{1}+a_{3}\right]^{\prime}$. Because

$$
b_{3} \mu\left(-a_{1}+a_{3}\right) \geqslant b_{1} \mu\left(-a_{1}+a_{3}\right)
$$

it follows that $b_{3} \nu\left(-a_{2}+a_{1}\right)^{+} \mu a \geqslant b_{1} \nu\left(-a_{2}+a_{1}\right)^{+} \mu a$, and a similar argument shows $b_{3} \nu\left(-a_{1}+a_{2}\right)^{+} \mu a \geqslant b_{2} \nu\left(-a_{1}+a_{2}\right)^{+} \mu a$. It remains to show

$$
b_{3} \mu\left(-a_{2}+a_{1}\right) \mu a \geqslant\left(b_{1} \vee b_{2}\right) \mu\left(-a_{2}+a_{1}\right) \mu a \text {. }
$$

Now $A\left(-a_{1}+a_{3}\right) \subseteq A\left(-a_{1}+a_{2}\right) \vee A\left(-a_{2}+a_{3}\right)$ and $A\left(-a_{1}+a_{3}\right) \subseteq A\left(-a_{1}+a_{2}\right)$ $\vee A\left(-a_{1}+a_{3}\right)$ imply $A\left(-a_{1}+a_{3}\right) \subseteq A\left(-a_{1}+a_{2}\right) \vee A(a)$, and similarly

$$
A\left(-a_{2}+a_{3}\right) \subseteq A\left(-a_{1}+a_{2}\right) \vee A(a) \text {. }
$$

Therefore

$$
\left[-a_{1}+a_{3}\right] \vee\left[-a_{2}+a_{3}\right] \subseteq\left[-a_{1}+a_{2}\right] \vee[a]
$$

or

$$
\left[-a_{1}+a_{2}\right]^{\prime} \cap[a]^{\prime} \subseteq\left[-a_{1}+a_{3}\right]^{\prime} \cap\left[-a_{2}+a_{3}\right]^{\prime}
$$


Then $b_{3} \mu\left(-a_{1}+a_{3}\right) \geqslant b_{1} \mu\left(-a_{1}+a_{3}\right)$ and $b_{3} \mu\left(-a_{2}+a_{3}\right) \geqslant b_{2} \mu\left(-a_{2}+a_{3}\right)$ imply

$$
b_{3} \mu\left(-a_{2}+a_{1}\right) \mu a \geqslant\left(b_{1} \vee b_{2}\right) \mu\left(-a_{2}+a_{1}\right) \mu a \text {. }
$$

Lemma 3.10. B lies below $A$ in $G$.

Proof. Given $0<(0, b) \in B$ and $0<(a, 0) \in A,(a, 0) \wedge(0, b)=(0, b \nu a)$, hence $n(a \wedge b)=(0, n b \nu a) \leqslant(a, 0)$ because $a \geqslant 0$ and $(n b \nu a) \mu a=0 \leqslant 0 \mu a$.

The reader may readily verify that $b \sigma(a)=-a+b+a$ and that $[a]=G(a) \cap B$ for each $a \in A$ and $b \in B$. Thus Lemma 3.10 completes the proof of Theorem 3.4. We shall refer to $G$ as the splitting extension of $B$ by $A$ determined by $\sigma$ and $\pi$. Because $A$ is obviously maximal among subgroups of $G$ lying above $B$ and likewise $B$ maximal below $A$, we know that $A$ and $B$ are closed $l$-subgroups of $G$, that $B$ is convex, and that $A$ is saturated.

The next few results concern $\mathscr{C}(G)$ as determined by $\mathscr{C}(A)$ and $\mathscr{C}(B)$. We continue to assume the terminology of Theorem 3.4.

Proposition 3.11. Let $C$ and $D$ be l-subgroups of $A$ and $B$, respectively. Then $C \times D$ is an l-subgroup of $G$ if and only if $D \sigma(c)=D$ and $D=(D \cap[c]) \boxplus\left(D \cap[c]^{\prime}\right)$ for all $c \in C$. In this case $C \times D$ is the splitting extension of $D$ by $C$ determined by (the restrictions of) $\sigma$ and $\pi$.

Proof. Clearly $C \times D$ is closed under addition if and only if $D \sigma(c)=D$ for all $c \in C$. Given $0<c \in C$ and $0<d \in D, c \wedge d=(0, d \nu c)$. Thus in order for $C \times D$ to be a sublattice it is necessary that $D=(D \cap[c]) \boxplus\left(D \cap[c]^{\prime}\right)$ for all $c \in C$; Lemma 3.9 shows that this condition is sufficient as well.

Given an $l$-subgroup $C$ of $A$ let $B_{C}=\bigcup\{[c]: c \in C\}$. Notice that $B_{C} \in \mathscr{C}(B)$ because $\{[c]: c \in C\}$ is a directed set. Given the $l$-subgroup $D$ of $B$ let $A_{D}=\{a \in A$ : $[a] \subseteq D\} \in \mathscr{C}(A)$.

Proposition 3.12. The following are equivalent for l-subgroups $C \leqslant A$ and $D \leqslant B$.

(a) $C \times D \in \mathscr{C}(G)$.

(b) $C \in \mathscr{C}(A)$ and $B_{C} \subseteq D \in \mathscr{C}(B)$.

(c) $D \in \mathscr{C}(B)$ and $A_{D} \supseteq C \in \mathscr{C}(A)$.

Furthermore, every $E \in \mathscr{C}(G)$ is of the form $E=C \times D$ for unique l-subgroups $C=E \cap A$ and $D=E \cap B$ satisfying the conditions above.

Proof. Suppose $C \times D \in \mathscr{C}(G)$. Then $0<x<c \in C$ implies $0<(x, 0)<(c, 0)$ $\in C \times D$, hence $(x, 0) \in C \times D$ or $x \in C \in \mathscr{C}(A)$. Likewise $D \in \mathscr{C}(B)$. For any $c \in C$ and $0<b \in[c],(0,0)<(0, b)<(|c|, 0) \in C \times D$, hence $(0, b) \in C \times D$ by convexity, or $b \in D$. This proves that (a) implies (b); now assume (b) to prove (a). For $c \in C, D=[c] \boxplus\left(D \cap[c]^{\prime}\right)$ and $D \sigma(c)=[c] \sigma(c) \boxplus\left(D \cap[c]^{\prime}\right)=D$, so $C \times D$ is an $l$-subgroup by Proposition 3.11. Consider $0<(x, y)<(c, d) \in C \times D$. We know from Lemma 3.8 that $0 \leqslant x \leqslant c, y \mu x \geqslant 0$, and $d \mu(-x+c) \geqslant y \mu(-x+c)$. It follows that $x \in C$ and that $d \mu c \geqslant y \mu c \geqslant 0$, and since $d \nu c \in[c] \subseteq D, d \mu c \in D$ and $y \mu c \in D$ by convexity. That is, $y=y \nu c+y \mu c \in D$, proving (a). (b) and (c) are clearly equivalent. 
Suppose $E \in \mathscr{C}(G)$ and let $C=E \cap A$ and $D=E \cap B$. Observe that $0<(x, y)$ $\in G$ implies $0 \leqslant(x, 0) \leqslant 2(x, y)=(2 x, y \sigma(x)+y)$ because $2 x \geqslant x$ and

$$
(y \sigma(x)+y) \mu(-x+2 x)=y \mu x \geqslant 0=0 \mu(-x+2 x) .
$$

Therefore $0<(c, d) \in E$ implies $(c, 0) \in E$ by convexity. If $(c, d)$ is an arbitrary element of $E$, then $(c, d)^{+}=\left(c^{+}, x\right) \in E$ and $(c, d)^{-}=\left(c^{-}, y\right) \in E$ for some $x$, $y \in B$, hence $\left(c^{+}, 0\right),\left(c^{-}, 0\right) \in E$ as above, and $(c, 0)=\left(c^{+}, 0\right)-\left(c^{-}, 0\right) \in E$ and $-(c, 0)+(c, d)=(0, d) \in E$, proving $E=C \times D$.

Proposition 3.13. The map $C \rightarrow C \times B$ is a lattice isomorphism from $\mathscr{C}(A)$ onto $\{E \in \mathscr{C}(G): B \subseteq E\}$. Thus $C$ is a prime subgroup of $A$ if and only if $C \times B$ is a prime subgroup of $G$. In particular, $C$ is a value of $a \in A$ if and only if $C \times B$ is a value of $(a, b) \in G$ for any $b \in G$. In this case $C$ is normal in its cover $C^{*}$ if and only if $C \times B$ is normal in its cover $C^{*} \times B$.

Proof. The first assertion follows from Proposition 3.12, the second follows from the fact that a convex $l$-subgroup is prime if and only if the convex $l$-subgroups containing it form a chain, and the third and fourth assertions can be verified in straightforward fashion.

The following lemma is well known and quite general. We prove it here for completeness.

LeMma 3.14. Suppose $B$ and $D$ are convex l-subgroups of an l-group $G$ and that $D \leqslant B$. Then $X=\left\{g \in G:|g| \wedge b \in D\right.$ for all $\left.b \in B^{+}\right\}$is the maximum element of $\{E \in \mathscr{C}(G): E \cap B \subseteq D\}$. X is prime in $G$ if and only if $D$ is prime in $B$.

Proof. If $0<x_{1}, x_{2} \in X$ and $0<b \in B$, then

$$
\begin{aligned}
0 & \leqslant b \wedge\left(x_{1}+x_{2}\right) \leqslant(2 b) \wedge\left(b+x_{2}\right) \wedge\left(x_{1}+b\right) \wedge\left(x_{1}+x_{2}\right) \\
& =b \wedge x_{1}+b \wedge x_{2} \in D .
\end{aligned}
$$

It follows that $X$ is a convex l-subgroup, and hence the maximum element of $\{E \in \mathscr{C}(G): E \cap B \subseteq D\}$. Suppose now that $D$ is prime in $B$, and that $u, v \in G$ with $u \wedge v=0$. If $u \notin X$, then there is some $b_{0} \in B^{+}$such that $u \wedge b_{0} \notin D$. Thus for any $b \in B^{+}, u \wedge\left(b \vee b_{0}\right) \geqslant u \wedge b_{0} \notin D$ and $\left[u \wedge\left(b \vee b_{0}\right)\right] \wedge\left[v \wedge\left(b \vee b_{0}\right)\right]$ $=(u \wedge v) \wedge\left(b \vee b_{0}\right)=0$ imply $v \wedge\left(b \vee b_{0}\right) \in D$, meaning $v \wedge b \in D$ or $v \in X$.

Given $B \in \mathscr{C}(G)$ let $\mathscr{C}_{B}(G)=\{X \in \mathscr{C}(G): X \subsetneq Y \in \mathscr{C}(G)$ implies $X \cap B \subsetneq Y$ $\cap B\}$.

Proposition 3.15. The map $D \rightarrow A_{D} \times D$ is a one-one order preserving map from $\mathscr{C}(B)$ onto $\mathscr{C}_{B}(G)$. Thus $D$ is a prime subgroup of $B$ if and only if $A_{D} \times D$ is a prime subgroup of $G$, and every prime subgroup $E$ of $G$ such that $B \nsubseteq E$ has the form $E=A_{D} \times D$ for a (unique) proper prime subgroup $D$ of $B$. For such $a D, A_{D} \times B$ is a value of $(a, b)$ in $G$ is and only if $D$ is a value of bua in $B$.

Proof. The fact that $A_{D} \times D$ is the maximum element of $\{E \in \mathscr{C}(G): E \cap B \subseteq$ $D$ \} follows from Proposition 3.12, and the second assertion results from Lemma 3.14. Suppose now that $E$ is a prime subgroup of $G$ for which $B \nsubseteq E$. By 
Proposition $3.12 E=C \times D$ for $D$ a proper prime subgroup of $B$ and $C \in \mathscr{C}(A)$ such that $C \leqslant A_{D}$. If $0<a \in A_{D} \backslash C$, then $a \notin E$, so $[a]^{\prime} \subseteq a^{\perp} \subseteq E$, hence $[a]^{\prime} \subseteq$ $E \cap B=D$. But together with the fact that $[a] \subseteq D$ since $a \in A_{D}$, this violates the assumption that $B \neq D$. We conclude that $C=A_{D}$ and $E=A_{D} \times D$.

Suppose that $D$ is a proper prime subgroup of $B$, and fix $a \in A$ and $b \in B$. If $D$ is a value of $b \mu a$ in $B$ with cover $D^{*}$, then $(a, b) \notin A_{D} \times D$, and by the preceding paragraph every $E \in \mathscr{C}(G)$ satisfying $A_{D} \times D \subsetneq E$ also satisfies $D^{*} \subseteq E$, hence $A_{D} \times D$ is a value of $(0, b \mu a)$ in $G$. Now $(0, b \mu a)$ is a component of $(a, b)$ (because $(a, b)=(a, b \nu a)+(0, b \mu a))$, so $A_{D} \times D$ is a value of $(a, b)$ as well. Now suppose $A_{D} \times D$ is a value of $(a, b)$. It follows that $a \in A_{D}$, for otherwise $A_{D} \times B$ is a prime subgroup properly containing $A_{D} \times D$ which nevertheless does not contain $(a, b)$. By convexity, $(a, b \nu a) \in A_{D} \times D$; hence $(0, b \mu a) \notin D$, and if $D \subseteq E \in \mathscr{C}(B)$, then $(0, b \mu a) \in A_{E} \times E \supsetneq A_{D} \times D$, meaning that $D$ is a value of $b \mu a$.

Proposition 3.16. If $D$ and $E$ are prime subgroups of $B$ such that $D \vee E \neq B$ in $\mathscr{C}(B)$, then $A_{D}=A_{E}$.

Proof. It is sufficient to establish the case in which $D \subsetneq E \subsetneq B$. Now $A_{E} \times E$ and $A_{D} \times B$ are members of $\mathscr{C}(G)$ containing the prime subgroup $A_{D} \times D$, and so are related. Since $B \nsubseteq E$ we must have $A_{E} \times E \subseteq A_{D} \times B$, or $A_{E} \subseteq A_{D}$.

Proposition 3.16 completes the description of $\Gamma(G)$ from $\Gamma(A)$ and $\Gamma(B)$. Call a pair of prime subgroups $D$ and $E$ of $B$ distantly related if $D \vee E \neq B$ in $\mathscr{C}(B)$ and let $\langle\langle E\rangle\rangle=\{D: E$ and $D$ are distantly related prime subgroups of $B\}$. Start with $\Gamma(A)$, and attach each equivalence class $\langle\langle E\rangle\rangle$ below $A_{E} \in \Gamma(A)$. (Actually, we are cheating a bit here. In this paragraph only are we considering $\Gamma(A)$ to be the partially ordered set of all (not only regular) primes of $A$.) The resulting partially ordered set is $\Gamma(G)$.

Corollary 3.17. $G$ is normal-valued if and only if both $A$ and $B$ are normal-valued.

Proof. Suppose that $A$ and $B$ are normal valued, that $P$ is a regular prime of $G$ with cover $P^{*}$, and that $(a, b) \in P^{*} \backslash P$. If $B \subseteq P$, then $P=C \times B$ for some value $C$ of $a$ in $A$ having cover $C^{*}$. For any $\left(c, b_{1}\right) \in P$,

$$
-(a, b)+\left(c, b_{1}\right)+(a, b)=\left(-a+c+a, b_{2}\right) \in C^{*} \times B=P^{*} .
$$

If $B \nsubseteq P$, then $P=A_{D} \times D$ for some value $D$ of $b \mu a$ in $B$ having value $D^{*}$. Since $b \mu a$ is a component of $(a, b),-(a, b)+P+(a, b)=-b \mu a+P+b \mu a$. So consider arbitrary $(c, d) \in P=A_{D} \times D$. Then

$$
\begin{aligned}
-(0, b \mu a)+(c, d)+(0, b \mu a) & =(c,-b \mu a \sigma(c)+d+b \mu a) \\
= & (c,-b \mu a \nu c \sigma(c)+(-b \mu a \mu c+d+b \mu a \mu c)+b \mu a \nu c),
\end{aligned}
$$

and $b \mu a \nu c \sigma(c)$ and $b \mu a \nu c$ lie in $D$ because $[c] \subseteq D$ since $c \in A_{D}$, while $(-b \mu a \mu c+$ $d+b \mu a \mu c) \in D$ because $b \mu a \mu c \in D^{*}$ since $b \mu a \in D^{*}$. Therefore $-(a, b)+P+$ $(a, b) \subseteq P^{*}$.

Here is an example which shows that $G$ need not lie in $\mathbf{S}$ when $A$ and $B$ do. Let $N$ be the trivially ordered set $\{0,1,2, \ldots\}$, let $B=\Sigma(N, \mathbf{Z})$, let $A=\{g \in V(N, \mathbf{Z})$ : there is some $m \in N$ such that $(n) g=(0) g$ for all $n \geqslant m\}$, and for each $a \in A$ let 
$\sigma(a)$ be the identity $l$-automorphism of $B$ and $\pi(a)=[a]=\{b \in B:(n) b \neq 0$ implies $(n) a \neq 0$ for all $n \in N\} \in \mathscr{S}(B)$. Let $a \in A$ be the element such that $(n) a=1$ for all $n \in N$. Then no disjoint set of special elements in $G$ can have supremum $a$.

Corollary 3.18. $G$ is finite-valued if and only if both $A$ and $B$ are.

Proof. The set of all values of $(a, b) \in G$ is $\{P \times B: P$ is a value of $a$ in $A\} \cup\left\{A_{D} \times D: D\right.$ is a value of $b \mu a$ in $\left.B\right\}$, a finite set.

We close this section by pointing out that the splitting extension developed in this section is general enough to encompass cardinal product, lex extension, and permutation wreath product.

THEOREM 3.19. Given l-groups $A$ and $B$ define $\pi(A(a))=[a]=0 \in \mathscr{S}(B)$ for each $a \in A$ and define $\sigma(a)$ to be the identity automorphism of $B$. Then the splitting extension of $B$ by $A$ determined by $\pi$ and $\sigma$ is (l-isomorphic to) the cardinal product $A \boxplus B$.

The notion of lex extension is developed in [10].

THEOREM 3.20. Suppose $\sigma$ is any group homomorphism from the totally ordered group $A$ into the l-automorphisms of the l-group $B$, and suppose $\pi(A(a))=[a]=B \in$ $\mathscr{S}(B)$ for all $a \in A$. Then the splitting extension of $B$ by $A$ determined by $\pi$ and $\sigma$ is $a$ lex extension of $B$ by $A$. Conversely, if $G$ is any l-group whose lex kernel $B$ splits, that is for which $G=A+B$ for some subgroup $A$, then $A$ is totally ordered and there is some homomorphism $\sigma$ such that $G$ is obtained from $A$ and $B$ as above.

We employ the permutation wreath product notation of Chapter 5 of [13]. $\Pi_{T} G=\{f: f: T \rightarrow G\}$ designates the cardinal product of copies of $G$ indexed by $T$. For $f \in \Pi_{T} G$ we designate the component of $f$ at index $t$ by $f_{t}$.

THEOREM 3.21. Let $(G, \Omega)$ and $(H, T)$ be transitive l-permutation groups and let $B=\Pi_{T} G$. For $h \in H$ define $[h]=\pi(H(h))=\left\{f \in B: \alpha f_{t}=\alpha\right.$ for all $\alpha \in \Omega$ and $t \in T$ such that th $=t\} \in \mathscr{S}(B)$, and define $f \sigma(h) \in B$ by $\alpha(f \sigma(h))_{t}=\alpha f_{s}$, where $s=t^{-1} \in T$. Then the splitting extension of $B$ by $H$ determined by $\sigma$ and $\pi$ is l-isomorphic to $\mathrm{G} \mathrm{Wr} H$.

4. Characterizing the lattice homomorphism $\pi: \mathscr{C} p(A) \rightarrow \mathscr{S}(B)$. In some important instances it is possible to completely characterize the maps $\pi: \mathscr{C} p(A) \rightarrow$ $\mathscr{S}(B)$ of Proposition 3.3. The basic insight is lattice theoretical in nature.

Proposition 4.1. Suppose that $D$ is a distributive lattice and $E$ is a complete Boolean algebra. If for each prime ideal $P$ of $D$ there is an element $e_{P} \in E$ satisfying property (a) below, then the map $\pi: D \rightarrow E$ defined by $\pi(d)=\bigvee\left\{e_{P}: d \notin P\right\}$ enjoys properties (b), (c), and (d) below. Conversely, suppose $\pi: D \rightarrow E$ satisfies (b), (c), and (d) and define $e_{P}=(\Lambda\{\pi(d): d \notin P\}) \wedge\left(\Lambda\left\{\pi(d)^{\perp}: d \in P\right\}\right)$ for $P$ a prime ideal of $D$. Then (a) holds, and $\pi(d)=\bigvee\left\{e_{P}: d \notin P\right\}$ for all $d \in D$.

(a) $e_{P} \wedge e_{Q}=0$ for distinct prime ideals $P$ and $Q$.

(b) $\pi$ is a lattice homomorphism. 
(c) $\pi(0)=0$.

(d) The complete subalgebra $F \subseteq E$ generated by $\pi(D)$ is atomic.

Proof. Suppose that for each prime ideal $P \subseteq D$ there is an element $e_{P} \in E$ satisfying (a), and define $\pi(d)=\bigvee\left\{e_{P}: d \notin P\right\}$. Let $A=\left\{e_{P}: P\right.$ a prime ideal of $D\} \cup\left\{\wedge\left\{e_{P}^{\perp}: P\right.\right.$ a prime ideal of $\left.\left.D\right\}\right\}$, a maximal pairwise disjoint subset of $E$, and let $F=\left\{\bigvee A_{0}: A_{0} \subseteq A\right\}$, the complete subalgebra of $E$ generated by $A$. Observe that $F$ is isomorphic to the Boolean algebra of subsets of $A$, and that $A$ is the set of atoms of $F$. Properties (b) and (c) are immediate consequences of these observations; to establish (d) we must show that $\pi(D)$ generates $F$. This is done by showing that $e_{P}=e$ for each prime ideal $P$ of $D$, where $e$ stands for $(\bigwedge\{\pi(d): d \notin P\}) \wedge$ $\left(\bigwedge\left\{\pi(d)^{\perp}: d \in P\right\}\right)$. Now $e_{P} \leqslant e$ because $e_{P}$ is an atom of $F$, and if $e_{P}<e$ it could only be because $e_{Q} \leqslant e$ for some prime ideal $Q \neq P$. But if $d \in Q \backslash P$, then $e_{Q} \leqslant \pi(d)^{\perp}$ while $e_{P} \leqslant \pi(d)$, and similarly for $d \in P \backslash Q$. This contradiction establishes that $e_{P} \in F$.

Suppose that $\pi: D \rightarrow E$ satisfies (b), (c), and (d) and define

$$
e_{P}=(\bigwedge\{\pi(d): d \notin P\}) \wedge\left(\bigwedge\left\{\pi(d)^{\perp}: d \in P\right\}\right)
$$

for each prime ideal $P$ of $D$. If $P$ and $Q$ are distinct prime ideals, then there is some $d \in D$ such that $d \in P \backslash Q$, say, and so $e_{P} \leqslant \pi(d)^{\perp}$ and $e_{Q} \leqslant \pi(d)$, proving (a). Now consider a particular $d \in D$ and let $e=\bigvee\left\{e_{P}: d \notin P\right\}$. Clearly $\pi(d) \geqslant e$; to show equality, consider an atom $a \in F$ such that $a \leqslant \pi(d)$. Let $Q=\left\{d^{\prime} \in D\right.$ : $\left.\pi\left(d^{\prime}\right) \ngtr d\right\}=\left\{d^{\prime} \in D: \pi\left(d^{\prime}\right)^{\perp} \geqslant a\right\}$, a prime ideal of $D$. It is straightforward to verify that $a \leqslant e_{Q} \leqslant e$, from which follows $\pi(d)=e$.

The prime subgroups of an l-group $A$ correspond to the prime ideals of the distributive lattice $\mathscr{C} \not(A)$. Therefore the following result is an application of the preceding one. In the following we use $[a]$ to designate $\pi(A(a))$.

THEOREM 4.2. Let $A$ and $B$ be l-groups. If to each prime subgroup $P$ of $A$ there corresponds a polar $B_{P} \in \mathscr{P}(B)$ satisfying (a) and (b) below, then the map $\pi$ : $\mathscr{C} p(A) \rightarrow \mathscr{S}(B)$ defined by $[a]=\bigvee\left\{B_{P}: a \notin P\right\}$ enjoys properties (c), (d), and (e). Conversely, suppose $\pi: \mathscr{C} p(A) \rightarrow \mathscr{S}(B)$ satisfies (c), (d), and (e), and for each prime subgroup $P$ of $A$ define $B_{P}=(\cap\{[a]: a \notin P\}) \cap\left(\cap\left\{[a]^{\prime}: a \in P\right\}\right)$. Then (a) and (b) hold, and $\pi(a)=\bigvee\left\{B_{P}: a \notin P\right\}$.

(a) $B_{P} \cap B_{Q}=0$ for distinct prime subgroups $P$ and $Q$.

(b) $\bigvee\left\{B_{P}: a \notin P\right\} \in \mathscr{S}(B)$ for all $a \in A$. (The supremum here is taken in $\mathscr{P}(B)$.)

(c) $\pi$ is a lattice homomorphism.

(d) $\pi(0)=0$.

(e) The complete subalgebra of $\mathscr{P}(B)$ generated by $\pi(\mathscr{C} \not(A))$ is atomic.

LEMMA 4.3. A complete subalgebra $S$ of a complete atomic Boolean algebra $T$ is itself atomic.

Proof: Let $A$ be the set of atoms of $T$, and for each $a \in A$ let $(a)=\wedge\{s \in S$ : $a \leqslant s\} \in S$. Then $B=\{(a): a \in A\}$ is a maximal pairwise disjoint subset of $T$, hence $\left\{\bigvee B_{0}: B_{0} \subseteq B\right\}$ is a complete Boolean subalgebra of $T$ contained in $S$. Since 
$s=\bigvee\{a \in A: a \leqslant s\}$, it follows that $s=\bigvee\{(a) \in B: a \leqslant s\}$ for all $s \in S$. Therefore $S$ is $\left\{\bigvee B_{0}: B_{0} \subseteq B\right\}$ and $B$ is the set of atoms of $S$.

Corollary 4.4. Suppose $A$ and $B$ are l-groups and that $B$ has a basis. Then any lattice homomorphism $\pi: \mathscr{C} p(A) \rightarrow \mathscr{S}(B)$ such that $\pi(0)=0$ is of the type described in Theorem 4.2 .

Proof. $B$ has a basis if and only if $\mathscr{P}(B)$ is atomic.

Corollary 4.5. Suppose $A$ and $B$ are l-groups such that $\Gamma(A)$ is finite. Then any lattice homomorphism $\pi: \mathscr{C} h(A) \rightarrow \mathscr{S}(B)$ such that $\pi(0)=0$ has the form described in Theorem 4.2.

Proof. $\Gamma(A)$ is finite if and only if $\mathscr{C} h(A)$ is finite, in which case $\pi(\mathscr{C} \not(A))$ generates a complete subalgebra of $\mathscr{P}(B)$ which is finite and so atomic.

COROLlaRY 4.6. Suppose $B$ is any l-group and $A=\sum \mathbf{R}_{\lambda}$ is a small cardinal sum of archimedean o-groups. Then any lattice homomorphism $\pi: \mathscr{C} h(A) \rightarrow \mathscr{S}(B)$ has the form described in Theorem 4.2 .

Proof. Each $\mathbf{R}_{\gamma}$ is an atom of $\mathscr{C} \not(A)$ in the sense that it is minimal in $\mathscr{C} p(A) \backslash\{0\}$, and each element $A(a) \in \mathscr{C} p(A)$ is a finite join of such atoms. Therefore $\left\{\pi\left(\mathbf{R}_{\gamma}\right): \pi\left(\mathbf{R}_{\gamma}\right)>0\right\}$ is the set of atoms of the complete subalgebra of $\mathscr{P}(B)$ generated by $\pi(\mathscr{C} \not(A))$.

Corollary 4.7. Suppose $A$ and $B$ are l-groups such that each positive element of $B$ exceeds a positive special element. Then every lattice homomorphism $\pi: \mathscr{C} h(A) \rightarrow$ $\mathscr{S}(B)$ such that $\pi(0)=0$ has the form described in Theorem 4.2.

Proof. Observe that for any special $b \in B^{+}$and $a \in A$, either $b \in[a]$ or $b \in[a]^{\prime}$. Let $(b)=(\cap\{[a]: a \in A$ and $b \in[a]\}) \cap\left(\cap\left\{[a]^{\prime}: a \in A\right.\right.$ and $\left.\left.b \in[a]^{\prime}\right\}\right)$, a nonzero element of $\mathscr{P}(B)$, and let $C=\{(b): 0<b \in B, b$ special $\}$. Since each element of $B$ exceeds a special element, $[a]=\bigvee\{(b) \in C: b \in[a]\}$ for each $a \in A$. Therefore the complete subalgebra of $\mathscr{P}(B)$ generated by $\pi(\mathscr{C} h(A))$ is atomic, and $C$ is the set of its atoms.

If $B$ is finite-valued, then the polars $B_{P}$ of Theorem 4.2 are themselves cardinal summands. To prove this it is necessary only to observe that $\mathscr{S}(B)$ is a complete subalgebra of $\mathscr{P}(B)$ in this case.

LeMma 4.8. If $G$ is finite-valued, then $\mathscr{S}(G)$ is a complete sublattice of $\mathscr{P}(G)$.

Proof. Observe that for special $t \in G^{+}$and $C \in \mathscr{S}(G), t \in C$ or $t \in C^{\perp}$. ( $t=c+d$ for $c \in C$ and $d \in C^{\perp}$, and the value $P$ of $t$ contains exactly one of $c$ and $d$, say $d$. For every prime subgroup $Q \subseteq P, c \notin P$ implies $(t-c)=d \in Q$, or $Q+t=Q+c$. For any prime $Q \nsubseteq P$ we get $t \in Q$, since some multiple of $q \in Q \backslash P$ will exceed $t$. Therefore $Q \leqslant Q+c \leqslant Q+t=Q$ in that case also. This proves $t=c$.) Suppose now that $\vee \mathscr{C}=D$ in $\mathscr{P}(B)$ for some $\mathscr{C} \subseteq \mathscr{S}(B)$, and consider $g \in G^{+}$. Then $g=g_{1}+g_{2}+\cdots+g_{n}$ for disjoint special elements $g_{i}$. By relabelling we may assume $g_{i} \in \cup \mathscr{C}$ for $1 \leqslant i \leqslant k$ and $g_{i} \notin \bigcup \mathscr{C}$ for $k<i \leqslant n$. 
Then $\left(g_{1}+g_{2}+\cdots+g_{k}\right) \in D$ and $\left(g_{k+1}+g_{k+2}+\cdots+g_{n}\right) \in \bigcap\left\{C^{\perp}: C \in \mathscr{C}\right\}$ $=D^{\perp}$.

Proposition 4.9. Suppose $A$ and $B$ are l-groups such that $B$ is finite-valued, and that $\pi: \mathscr{C} h(A) \rightarrow \mathscr{S}(B)$ is a lattice homomorphism with $\pi(0)=0$. Then for each prime $P$ of $A$ there is a summand $B_{P} \in \mathscr{S}(B)$ such that $B_{P} \neq B_{Q}$ for distinct primes $P$ and $Q$, and such that $[a]=\sum\left\{B_{P}: a \notin P\right\}$ for each $a \in A$.

The following theorem summarizes the results of this investigation as applied to lattice orderings of semidirect products of l-groups in which the factors appear above and below one another.

THEOREM 4.10. Suppose $A$ and $B$ are l-groups, and suppose the group semidirect product $G=A \times B$ is endowed with a lattice order extending the orders on $A$ and $B$. If $A$ lies above $B$, then $A$ is maximal above $B$ and hence is saturated, and $B$ is maximal below $A$ and hence convex. Conversely, if $A$ is saturated, $B$ is convex, and if $B$ satisfies (a) or (b) below, then $A$ lies above B. Furthermore, if $A$ lies above $B$ and any one of (c), (d), (e), or (f) holds, then the lattice order on $G$ is determined as per Theorem 4.2.

(a) $B=\bigvee(T \wedge b)$ for all $b \in B^{+}$, where $T=\left\{t \in B^{+}: t\right.$ special $\}$.

(b) $\mathscr{S}(B)$ is dense in $\mathscr{P}(B)$.

(c) $B$ has a basis.

(d) $\Gamma(A)$ is finite.

(e) $A$ is a cardinal sum of archimedean o-groups.

(f) Each positive element of $B$ exceeds a positive special element.

Proof. The subgroup $A$ is maximal with respect to $A \cap B=0$, and so is maximal above $B$ whenever it lies above $B$. In this case $A$ must be saturated and $B$ convex by Theorem 1.6. The converse is Proposition 1.12. The last assertion has been established in the preceding corollaries.

As an example of Theorem 4.10 consider an l-group $G$ which is a splitting extension of the $l$-ideal $B$ by the saturated $l$-subgroup $A$, where $B$ is $l$-isomorphic to $\sum_{i=1}^{n} \mathbf{Z}_{i}$ and $A$ is divisible. Since the group of $l$-automorphisms of $B$ is the group of permutations on $n$ elements and since any homomorphic image of $A$ is divisible, $G$ is the group direct product of $A$ and $B$. Furthermore, $B$ lies below $A$, and the order on $G$ is given by assigning a cardinal summand of $B$ to each prime of $A$ as in Theorem 4.2.

5. l-groups in which each maximal below subgroup splits. Let $\mathbf{S}_{1}\left(\mathbf{S}_{2}\right)$ be the class of l-groups $G$ such that if $B$ is a maximal below subgroup of $G$, then $G=A+B$ for some subgroup (every subgroup) $A$ maximal above $B$. We shall show that these classes are closed with respect to convex $l$-subgroups, cardinal sums, and cardinal products.

Lemma 5.1. Suppose $(A, B)$ is a maximal above and below pair of $G=G_{1} \boxplus G_{2}$. Then $B=\left(B \cap G_{1}\right) \boxplus\left(B \cap G_{2}\right)$ and $A=\left(A \cap G_{1}\right) \boxplus\left(A \cap G_{2}\right)$, so $\left(A \cap G_{i}, B \cap\right.$ $\left.G_{i}\right)$ is a maximal above and below pair in $G_{i}$ for $i=1,2$. 
Proof. $0<b \in B$ implies $b=b_{1}+b_{2}$ for unique $b_{i} \in G_{i}$, and $b_{1}, b_{2} \geqslant 0$ because the order on $G$ is the lattice product of the orders on the $G_{i}$ 's. Therefore $b_{1}$, $b_{2} \in B$ by convexity, meaning $B=\left(B \cap G_{1}\right) \boxplus\left(B \cap G_{2}\right)$. Likewise $0<a \in A$ implies $a=a_{1}+a_{2}$ for unique $a_{i} \in G_{i}$, and the $a_{i}$ 's are components of $a$, again by virtue of the order on $G$. Then $a_{1}, a_{2} \in A$ because $A$ is saturated by Theorem 1.6, so that $A=\left(A \cap G_{1}\right) \boxplus\left(A \cap G_{2}\right)$.

It is clear from Proposition 1.4 that $A \cap G_{1}$ lies below $B \cap G_{1}$ in $G_{1}$. If $C$ is maximal among those convex $l$-subgroups of $G_{1}$ which contain $A \cap G_{1}$ and lie below $B \cap G_{1}$, then $C \boxplus\left(B \cap G_{2}\right)$ lies below $A$, hence $C=A \cap G_{1}$. A similar argument shows that $A \cap G_{1}$ is maximal among $l$-subgroups of $G_{1}$ above $B \cap G_{1}$.

Proposition 5.2. If $G \in \mathbf{S}_{n}$ and $C \in \mathscr{C}(G)$, then $C \in \mathbf{S}_{n}, n=1,2$.

Proof. Suppose $G \in \mathbf{S}_{1}, C \in \mathscr{C}(G)$, and $B$ is maximal below $A$ in $C$. Find $B_{1} \in \mathscr{C}(G)$ maximal among those convex $l$-subgroups of $G$ containing $B$ and lying below $A$. Note that $B_{1} \cap C$ lies below $A$ in $C$, hence $B_{1} \cap C=B$. Now find $A_{1}$ maximal among $l$-subgroups of $G$ above $B_{1}$ such that $G=A_{1}+B_{1}$. By Proposition $3.12 C=\left(A_{1} \cap C\right)+B$, proving $C \in \mathbf{S}_{1}$. The argument for $G \in \mathbf{S}_{2}$ is similar.

Proposition 5.3. The following are equivalent for any set $\left\{G_{\lambda}: \lambda \in \Lambda\right\}$ of l-groups.

(a) $G_{\lambda} \in \mathbf{S}_{1}$ for all $\lambda \in \Lambda$.

(b) $\sum G_{\lambda} \in \mathbf{S}_{1}$.

(c) $\Pi G_{\lambda} \in \mathbf{S}_{1}$.

These conditions remain equivalent if $\mathbf{S}_{1}$ is replaced by $\mathbf{S}_{2}$.

Proof. Proposition 5.2 provides the implication from (c) to (b) to (a). Suppose that $G_{\lambda} \in \mathbf{S}_{1}$ for all $\lambda \in \Lambda$ and let $B$ be maximal below $A$ in $\Pi G_{\lambda}$. We may without loss of generality assume that $A$ is maximal above $B$, so that by Lemma $5.1 B \cap G_{\lambda}$ is maximal below $A \cap G_{\lambda}$ in $G_{\lambda}$ for each $\lambda \in \Lambda$. For each $\lambda \in \Lambda$ choose $A_{\lambda}$ maximal above $B \cap G_{\lambda}$ such that $G_{\lambda}=\left(B \cap G_{\lambda}\right)+A_{\lambda}$, and let $A_{1}=\left\{f \in \Pi A_{\lambda}\right.$ : $\lambda f \in A_{\lambda}$ for all $\left.\lambda \in \Lambda\right\}$. The componentwise nature of the operations on $\Pi G_{\lambda}$ makes it clear that $A_{1}$ lies above $B$ and that $A_{1}+B=\Pi G_{\lambda}$. The argument for $\mathbf{S}_{2}$ is similar.

Proposition 5.4. If $G$ is an archimedean l-group, then $G \in \mathbf{S}_{2}$ if and only if $G \in \mathbf{S}_{1}$ if and only if $G$ is projectable.

Proof. In an archimedean $l$-group the only maximal above and below pairs are complementary polars.

The most general condition sufficient for membership in $\mathbf{S}_{2}$ is given by Theorem 6.18 below, which we state here. Its proof in $\$ 6$ depends on none of the intervening material.

THEOREM 5.5. A divisible abelian sup $\mathscr{Y}$ complete l-group lies in $\mathbf{S}_{2}$.

In the following proposition let $F(\Gamma, \mathbf{R})$ denote $\{g \in V(\Gamma, \mathbf{R}):\{\gamma \in \Gamma:(\gamma) g \neq 0\}$ admits only finite disjoint subsets $\}[9]$. Observe that $\Sigma \leqslant F \leqslant V$. 
Proposition 5.6. For any root system $\Gamma, \Sigma(\Gamma, \mathbf{R}) \in \mathbf{S}_{1}, \quad F(\Gamma, \mathbf{R}) \in \mathbf{S}_{1}$, and $V(\Gamma, \mathbf{R}) \in \mathbf{S}_{2}$; furthermore $\Sigma(\Gamma, \mathbf{R}) \in \mathbf{S}_{2}$ and $F(\Gamma, \mathbf{R}) \in \mathbf{S}_{2}$ whenever $\Gamma$ satisfies the DCC.

Proof. The fact that $V(\Gamma, \mathbf{R}) \in \mathbf{S}_{2}$ is a consequence of Theorem 5.5. To see that $\Sigma(\Gamma, \mathbf{R}) \in \mathbf{S}_{1}$ consider $B \in \mathscr{C}(\Sigma)$. Let $A=\{g \in \Sigma: \gamma g=0$ for all $\gamma \in \Gamma(B)\}$, an $l$-subgroup which lies above $B$ by Proposition 1.4. Since $B=\{g \in \Sigma: \gamma g=0$ for all $\gamma \notin \Gamma(B)\}, A+B=\Sigma$. The proof that $F \in \mathbf{S}_{1}$ is analogous. Now suppose $\Gamma$ has the DCC and that $(A, B)$ is a maximal above and below pair in $F$. (The argument that $\Sigma \in \mathbf{S}_{2}$ is analogous.) Suppose for contradiction that $g \in F \backslash(A+B)$. At least one of the (finitely many) special components of $g$-say $g_{1}$-fails to lie in $A+B$. Let $\gamma_{1}$ be the value of $g_{1}$. Corollary 1.11 provides $a \in A$ such that $g_{1}-a$ has a value in $\Gamma(B)$. This implies that one of the special components of $a$-say $a_{1}$ - has value $\gamma_{1}$ and that $\left(\gamma_{1}\right) a_{1}=\left(\gamma_{1}\right) g_{1}$. Now $a_{1} \in A$ because $A$ is saturated, so $g_{1}-a_{1} \notin A+B$. Let $g_{2}$ be one of the special components of $g_{1}-a_{1}$ such that $g_{2} \notin A+B$, and let $\gamma_{2}$ be its value. Observe that $\gamma_{2}<\gamma_{1}$. But a continuation of this argument produces an infinite descending chain in $\Gamma$, contrary to the DCC.

The following example makes several points, the first being that $\Sigma(\Gamma, \mathbf{R})$ need not lie in $\mathbf{S}_{2}$ in general. Let $\Gamma$ be $\Delta \cup \Lambda$, where $\Delta(\Lambda)=\left\{0^{n}\left(0^{n} 1\right): n=1,2,3, \ldots\right\}$. Here $0^{n}\left(0^{n} 1\right)$ refers to the string of $n$ zeros (followed by a single 1$)$. Order $\Gamma$ by declaring $\alpha>\beta$ whenever $\beta=\alpha \gamma$ for some $\gamma \in \Gamma$ (where $\alpha \gamma$ is the string formed by concatenating the $\alpha$ and $\gamma$ strings). Let $B=\{g \in \Sigma(\Gamma, \mathbf{R}): \Gamma(g) \subseteq \Lambda\}$ and define $a_{n} \in \Sigma$ by

$$
(\gamma) a_{n}= \begin{cases}1 & \text { if } \gamma=0^{n}, 0^{n} 1, \text { or } 0^{n+1} \\ 0 & \text { otherwise. }\end{cases}
$$

Let $A$ be the $l$-subspace of the vector lattice $\Sigma$ generated by $\left\{a_{n}: n=1,2,3, \ldots\right\}$. Then $A$ lies above $B$ by Proposition 1.4 since $\Gamma(A) \subseteq \Delta$, and in fact $(A, B)$ is a maximal above and below pair by Corollary 1.11 . Yet $A+B$ is proper in $\Sigma$, since the latter fails to contain the element $g \in \Sigma$ defined by

$$
(\gamma) g= \begin{cases}1 & \text { if } \gamma=0 \\ 0 & \text { otherwise }\end{cases}
$$

A finite-valued vector lattice need not belong to $\mathbf{S}_{1}$. Let $\Gamma$ have the meaning above. In his thesis [8], Byrd showed that $\Sigma(\Gamma, \mathbf{R})$ has an uncountable number of pairwise non-l-isomorphic $a$-closures in the class of abelian $l$-groups, each of which is a vector lattice $G$ which may be taken to satisfy $\Sigma(\Gamma, \mathbf{R}) \leqslant G \leqslant V(\Gamma, \mathbf{R})$. (See Chapter 4 of [9] for additional background.) We will show that exactly one of these $a$-closures lies in $\mathbf{S}_{1}$. Now if $B$ has the meaning above then it is still maximal below $A$ in $G$; therefore if $G \in \mathbf{S}_{1}$ then there must be some $l$-subgroup $A_{1}$ above $B$ in $G$ such that $G=A_{1}+B$. The map $\theta: A_{1} \rightarrow V(\Gamma, \mathbf{R})$ defined by

$$
(\gamma) \theta(a)= \begin{cases}(\gamma) a & \text { if } \gamma \in \Delta, \\ 0 & \text { if } \gamma \in \Lambda\end{cases}
$$


is an $l$-monomorphism and so it can be extended to an $l$-monomorphism $\hat{\theta}: G \rightarrow V$, which must be the identity on $B$. (See Theorem 3.3 of [12].) Since $G \hat{\theta} \leqslant F(\Gamma, \mathbf{R})=$ $\{g \in V:\{\gamma \in \Lambda:(\gamma) g \neq 0\}$ finite $\}$ and since $F$ is an $a$-extension of $\Sigma$, it follows that $G$ is $l$-isomorphic to $F$.

Corollary 5.7. A finite-valued vector lattice $G$ of countable dimension belongs to $\mathbf{S}_{1}$.

Proof. $G$ is $l$-isomorphic to $\Sigma(\Gamma, \mathbf{R})$ for a suitable choice of $\Gamma$ [9].

Proposition 5.8. A totally ordered group $G$ belongs to $\mathbf{S}_{1}$ if and only if each convex subgroup splits. That is, for each $B \in \mathscr{C}(G)$ there is some subgroup $A$ such that $A+B=G$.

Proof. Suppose $G \in \mathbf{S}_{1}$. Each regular subgroup of $G$ is maximal below by Proposition 2.3 and so splits. In particular, each regular subgroup and hence each convex subgroup is normal. Given $B \in \mathscr{C}(G)$ choose $a_{\gamma} \in G^{\gamma} \backslash G_{\gamma}$ for each regular $G_{\gamma}$ such that $G_{\gamma} \supseteq B$. Then $B$ is maximal below the subgroup $A$ generated by the $a_{\gamma}$ 's. The converse is obvious.

Proposition 5.9. Suppose $G \in \mathbf{S}_{2}$. If $\left(A_{1}, B\right)$ and $\left(A_{2}, B\right)$ are maximal above and below pairs in $G$, then $A_{1}$ is l-isomorphic to $A_{2}$.

Proof. Both $A_{1}$ and $A_{2}$ are $l$-isomorphic to $G / B$.

Does Proposition 5.9 hold even when $G$ does not lie in $\mathbf{S}_{2}$ ? The authors know of no counterexample to this conjecture. However, the following example provides counterexamples to several closely related conjectures. Let $\Gamma=\left\{a_{1}, a_{2}, b_{1}, b_{2}\right\}$, ordered by declaring $a_{i}>b_{i}$, and let $B=V(\Gamma, \mathbf{Z})$. Let $A$ be $\mathbf{Z}, \pi(A(a))=[a]=B$ $\in \mathscr{S}(B)$ for all $0 \neq a \in A$, and let $\sigma(1)$ be the $l$-automorphism of $B$ defined by $(x)(g \sigma(1))=(\psi(x)) g$, where $\psi\left(a_{1}\right)=a_{2}, \psi\left(a_{2}\right)=a_{1}, \psi\left(b_{1}\right)=b_{2}$, and $\psi\left(b_{2}\right)=b_{1}$. Let $G$ be the splitting extension of $B$ by $A$ determined by $\pi$ and $\sigma$. $G$ is a Scrimger group [15] and so normal-valued, and its elements can be naturally represented in the form

$$
\left(\begin{array}{lll} 
& m & \\
n_{1} & & n_{2} \\
p_{1} & & p_{2}
\end{array}\right)
$$

for integers $m, n_{i}$, and $p_{i}$. Using this notation let $A_{1}=\left\{g \in G: m=n_{1}=p_{1}=p_{2}\right.$ $=0\}, A_{2}=\left\{g \in G: m\right.$ even, $\left.n_{1}=p_{1}=p_{2}=0\right\}, A_{3}=\left\{g \in G: p_{1}=p_{2}=0\right\}$, $B_{1}=\left\{g \in G: m=n_{1}=n_{2}=p_{2}=0\right\}, B_{2}=\left\{g \in G: m=n_{2}=0\right\}$, and $B_{3}=\{g$ $\left.\in G: m=n_{1}=n_{2}=0\right\}$. Observe that $B_{1}$ lies below $A_{1}$, that $B_{2}$ is maximal with respect to containing $B_{1}$ and lying below $A_{1}$, that $A_{2}$ is maximal with respect to containing $A_{1}$ and lying above $B_{2}$, that $A_{3}$ is maximal with respect to containing $A_{1}$ and lying above $B_{1}$, and that $B_{3}$ is maximal with respect to containing $B_{1}$ and lying below $A_{3}$. The point is that $A_{2}$ is not $l$-isomorphic to $A_{3}, B_{2}$ is not $l$-isomorphic to $B_{3}$, and that $A_{2}+B_{2}$ is not $l$-isomorphic to $A_{3}+B_{3}$. 
6. Abelian l-groups $G$ which split off from below in every abelian extension in which $G$ is a convex $l$-subgroup. Suppose $G$ is a convex $l$-subgroup of the $l$-group $H$. We say $G$ splits off from below in $H$ if $H=U+G$ for every $l$-subgroup $U \leqslant H$ such that $U$ is maximal above $G$. The subject of this section is a characterization of those abelian $l$-groups $G$ which split off from below in every abelian $l$-group having $G$ as a convex $l$-subgroup (Theorem 6.16). If $G$ has this property we shall sometimes simply say that $G$ splits off from below. The following lemma is useful; it is Lemma 5.6 of $[2]$.

Lemma 6.1. Suppose $X$ is a subset and $g$ an element of $G$ such that $g \geqslant X$. Then $X$ is type $\mathscr{Y}$ and $\mathrm{V} X=y$ if and only if $\mathrm{V}\left\{(y-x)^{\perp}: x \in X\right\}=1_{\mathscr{P}}$.

An $l$-group $G$ which splits off from below is closed in every $l$-group $H$ in which $G$ is a convex $l$-subgroup. The latter property depends on the possession by $G$ of a supremum for each subset of the following type. Let us call a subset $X \subseteq G^{+}$type $\mathscr{Z}$ if $X$ is type $\mathscr{Y}$ and if $\mathrm{V}(X \wedge g)$ exists in $G$ for all $g \in G . G$ is sup $\mathscr{Z}$ complete if every type $\mathscr{Z}$ subset of $G$ possesses a supremum in $G$.

Proposition 6.2. If $G$ is sup $\mathscr{Z}$ complete, then $G$ is closed in every l-group $H$ in which $G$ is a convex l-subgroup.

Proof. Suppose $\vee X=h \in H$ for $X=G^{+} \wedge h \subseteq G^{+}$. We first claim that $X$ is a type $\mathscr{Y}$ subset of $H^{+}$. Lemma 6.1 is enough to show $\bigvee_{x \in X}(h-x)^{\perp}=1_{\mathscr{P}}$ in the Boolean algebra $\mathscr{P}$ of polars of $H$. For this purpose consider $0<g \in H$; we shall prove that $g \notin(h-x)^{\perp \perp}$ for some $x \in X$. Since $G^{\perp \perp}$ is closed in $H$ it follows that $h \in G^{\perp \perp}$, which allows us to consider only the case in which $g \in G$. Because $\mathrm{V} X=h$, there must be some $x \in X$ with $x-g+h$ or $g+x \nless h$. Let $x^{\prime}=(g+$ $x) \wedge h \in X$; we claim $g \notin\left(h-x^{\prime}\right)^{\perp \perp}$. For $h \vee(g+x)>h$ implies $0<(h \vee(g$ $+x))-h=0 \vee(g+x-h)=g^{\prime} \leqslant g$, and $\left(h-x^{\prime}\right) \wedge g^{\prime}=[0 \vee(h-x-g)] \wedge$ $[0 \vee(g+x-h)]=0 \vee(-|h-x-g|)=0$. This completes the proof that $X$ is a type $\mathscr{Y}$ subset of $H$. Since $G$ is convex in $H$, intersection provides a one-one correspondence from $\left\{P \in \mathscr{P} \mid P \subseteq G^{\perp \perp}\right\}$ onto the set of polars of $G$, from which easily follows that $X$ is a type $\mathscr{Y}$ subset of $G$. $X$ is actually type $\mathscr{Z}$, since by convexity $\vee(X \wedge g)=(\vee X) \wedge g=h \wedge g \in G$ for any $g \in G$. Since $G$ is sup $\mathscr{Z}$ complete there is some $t$ such that $\vee X=t \in G$. But then $0 \leqslant h \leqslant t$ implies by convexity that $h \in G$.

COROllaRY 6.3. If $G$ is sup $\mathscr{Y}$ complete, then it is closed in any l-group in which it is a convex l-subgroup.

THEOREM 6.4. An abelian l-group $G$ is closed in every l-group in which it is a convex l-subgroup if and only if it is sup $\mathscr{Z}$ complete.

Proof. Let $G_{\mathscr{O}}$ be the sup $\mathscr{Y}$ completion of $G[1]$ and suppose $X \subseteq G^{+}$is a type $\mathscr{Z}$ set with $\vee X=t \in G_{\mathscr{y}} \backslash G$, and let $W$ be the $l$-subgroup of $G_{\mathscr{y}}$ generated by $G$ and $t$. We shall prove that $G$ is convex but not order closed in $W$. For this purpose we shall adopt the following terminology for this proof and the next one only. An element $s \in G$ will be admissible if $s \pi 0 g \in G$ for all $0<g \in G$, where $\pi a b: G_{\mathscr{Y}} \rightarrow[a, b]$ is 
the lattice homomorphism defined by $x \pi a b=(x \vee a) \wedge b$. Observe that $G$ is sup $\mathscr{Z}$ complete if and only if it contains each admissible element of $G_{\mathscr{g} y}$. However, the set of admissible elements is not closed under addition in general.

The first claim is that if $0<u, v \in G_{o y}$ are admissible, then so is $u+v$. This is true because

$$
\begin{aligned}
(u+v) \pi 0 g & =(u+v) \wedge g=(u+v) \wedge(g+v) \wedge(u+g) \wedge(g+g) \wedge g \\
& =(u \wedge g+v \wedge g) \wedge g=(u \pi 0 g+v \pi 0 g) \pi 0 g \in G .
\end{aligned}
$$

Therefore $n t$ is admissible for all positive integers $n$. The second claim is that if $0<u \in G_{\mathscr{Y}}$ is admissible, then $u \pi a b \in G$ for all $a<b$ in $G$. This is true because

$$
u \pi a b=(u \pi a b)^{+}+(u \pi a b)^{-}=u \pi a^{+} b^{+}+b^{-}=u \pi 0 b^{+} \vee a^{+}+b^{-} \in G .
$$

The third claim is that if $0<u \in G_{o y}$ is admissible, then $(k+u) \pi a b \in G$ for all $k$, $a, b \in G$ with $a<b$. This holds because

$$
(k+u) \pi a b=k+[(u \vee(-k \vee a)) \wedge(-k \vee b)],
$$

the latter term being in $G$ by the second claim. The fourth and final claim is that if $0<u \in G_{o y}$ is admissible, then

$$
(k-u) \pi a b=(-(-k+u)) \pi a b=-[(-k+u) \pi(-b)(-a)] \in G
$$

for all $k, a, b \in G$ with $a<b$.

To complete the proof of the theorem consider an arbitrary $w \in W$. $w$ has the form $\bigvee_{i=1}^{n} \wedge_{j=1}^{m}\left(k_{i j}+n_{i j} t\right)$ for $k_{i j} \in G$ and integers $n_{i j}$. The claims show each $k_{i j}+n_{i j} t$ to be admissible, and hence $w$ is admissible. Therefore $G$ is convex in $W$. However, $G$ is clearly not order closed because $\bigvee X=t \in W \backslash G$.

COROLlaRY 6.5. Given any abelian l-group $G$ there is an abelian l-group $H$ in which $G$ is an order dense convex l-subgroup and such that $H$ is closed in any l-group containing $H$ as a convex l-subgroup.

Proof. Let $G_{0}=G$. Having defined $G_{\alpha}$, define $G_{\alpha+1}$ to be $G_{\alpha}$ if $G_{\alpha}$ is sup $\mathscr{Z}$ complete, otherwise let $G_{\alpha+1}$ be an $l$-subgroup of $G_{\mathscr{y}}$ generated by $G_{\alpha}$ and some admissible $0<t \in G_{\mathscr{y}} \backslash G$. Take unions at limit ordinals, and let $H=G_{\alpha}$ for the least ordinal $\alpha$ such that $G_{\alpha}=G_{\alpha+1}$. The proof of Theorem 6.4 shows that $G$ is a convex $l$-subgroup of $H$.

It seems quite unlikely that the $l$-group $H$ of the preceding corollary is unique. The general question is open: which $l$-groups admit unique $\mathscr{Z}$ hulls?

Those abelian $l$-groups $G$ which split off from below in every abelian l-group having $G$ as a convex $l$-subgroup can be characterized by the possession of three properties (Theorem 6.16). One of these properties is divisibility.

Proposition 6.6. If an abelian l-group $G$ splits off from below in every lex extension, then $G$ is divisible.

Proof. Let $G^{d}$ be the divisible hull of $G$ and suppose $p$ is the least positive integer such that $p x \in G$ for some $0<x \in G^{d} \backslash G$. Consider the lexicographic product $\mathbf{Z} \overrightarrow{\times} G^{d}$ determined by letting $\sigma(n)$ be the identity $l$-automorphism on $G^{d}$ 
and $\pi(n)=[n]=G^{d}$ for each $n \in \mathbf{Z}$. Let $H$ be the $l$-subgroup consisting of all pairs of the form ( $p i+j, a+j x$ ), where $i, j \in \mathbf{Z}$ and $a \in G$. Such pairs form an $l$-subgroup because $(p i+j, a+j x) \vee(0,0)$ is either $(p i+j, a+j x)$ in case $p i+j$ $>0$, or is $(0,0)$ in case $p i+j<0$, or is $(0,(a-i(p x)) \vee 0)$ in case $p i+j=0$. $H$ has (a copy of) $G$ as the convex $l$-subgroup $0+G$. Let $U=\{(p i, a) \mid i \in \mathbf{Z}\}$, an $l$-subgroup of $H$ above $G$. To show that $U$ is maximal above $G$ consider an arbitrary $0<h=(p i+j, a+j x) \in H \backslash U$ and let $V$ be the $l$-subgroup of $H$ generated by $U$ and $h$. We may assume $0 \leqslant j<p$, and by subtracting $(p i, 0) \in U$ we may assume $h=(j, a+j x)$. If $j=0$ then $a \neq 0$, so $h$ itself has a value in $\Gamma(G)$, and if $j>0$ then $p h-(p j, 0)=(0, p a+j(p x)) \in V$ which also has a value in $\Gamma(G)$ since $0 \neq p a+j(p x) \in G$. This proves $U$ maximal above $G$, which proves the theorem since $U+G$ fails to contain $(1, x)$, among others.

Another characteristic of an abelian $l$-group $G$ which splits off from below is the summand sewing property or SSP. Given a collection $\mathscr{S}$ of summands of $G$, a map $\theta$ : $\mathscr{S} \rightarrow G$ is said to be consistent if $S \theta-R \theta \in(S \cap R)^{\perp}$ for all $S, R \in \mathscr{S}$. G has the summand sewing property if for every consistent $\theta: \mathscr{S} \rightarrow G$ there is some $g \in G$ such that $g-S \theta \in S^{\perp}$ for all $S \in \mathscr{S}$. Observe that such $g \in G$ must always exist for finite $\mathscr{S}$. Also note that $g$ need not be unique. Finally, observe that it is enough to consider consistent maps $\theta: \mathscr{S} \rightarrow G^{+}$.

The definition of the SSP just given bears close resemblance to the definition of orthocompleteness given in [2], and in fact this resemblance can be converted with little difficulty to a proof that orthocompleteness implies the SSP. Thus every l-group is order dense in an l-group which enjoys the SSP.

Proposition 6.7. If $G$ is sup $\mathscr{Y}$ complete, then $G$ has the SSP.

Proof. Suppose $\theta: \mathscr{S} \rightarrow G^{+}$is a consistent map. For each $S \in \mathscr{S}$ let $g_{S} \in G$ be the projection of $S \theta$ on $S$, and let $X$ be $\left\{g_{S} \mid S \in \mathscr{S}\right\}$. The fact that $\left(X-g_{S}\right)^{+}$ $\cup\left(-g_{S}+X\right)^{+} \subseteq S^{\perp}$ for all $S \in \mathscr{S}$ demonstrates that $X$ is a type $\mathscr{Y}$ set and that $\vee X=g$ exists in $G$. But it can readily be shown that $g-S \theta \in S^{\perp}$ for all $S \in \mathscr{S}$.

Proposition 6.8. An abelian l-group $G$ which splits off from below in every abelian l-group in which it is maximal below must have the SSP.

Proof. Let $\mathscr{S}=\left\{S_{\lambda}: \lambda \in \Lambda\right\}$ be a set of summands of $G$ and $\theta: \mathscr{S} \rightarrow G^{+}$a consistent map which denies the SSP. Let $\bar{G}$ be the orthocompletion of $G$ and choose $t \in \bar{G}$ such that $t-S_{\lambda} \theta \in S_{\lambda}{ }^{\perp}$ for all $\lambda \in \Lambda$. Let $\Pi \mathbf{Z}_{\lambda}$ and $\Sigma \mathbf{Z}_{\lambda}$ designate as usual the large and small cardinal products of the integers $\mathbf{Z}$ over the index set $\Lambda$. Designate by $c \in \Pi \mathbf{Z}$ the element defined by $\lambda c=1$ for all $\lambda \in \Lambda$, and let $A=\{n c+s \in \Pi \mathbf{Z}: n \in \mathbf{Z}, s \in \Sigma \mathbf{Z}\}$, an $l$-subgroup of $\Pi \mathbf{Z}$. Define $\pi: \mathscr{C} p(A) \rightarrow$ $\mathscr{S}(\bar{G})$ by declaring $\pi(A(a))=[a]=\bigvee\left\{\bar{S}_{\lambda}: \lambda \in \operatorname{supp}(a)\right\}$, where $\bar{S}_{\lambda}=S_{\lambda}{ }^{\perp} \in$ $\mathscr{S}(\bar{G})$. Observe that for any $a \in A, \operatorname{supp}(a)$ is a finite or cofinite subset of $\Lambda$ and so $\mathrm{V}\left\{\bar{S}_{\lambda}: \lambda \in \operatorname{supp}(a)\right\}$ exists in $\mathscr{S}(\bar{G})$. Let $\sigma$ be the map which assigns the identity $l$-automorphism of $\bar{G}$ to each $a \in A$, and let $K$ be the splitting extension of $\bar{G}$ by $A$ determined by $\sigma$ and $\pi$. Finally, let $H$ be $\{(n c+s, n t+g) \in K: n \in \mathbf{Z}, s \in \Sigma \mathbf{Z}$, $g \in G\}$. 
We first claim that $H$ is an $l$-subgroup of $K$. To verify this claim we must succeed in showing $(n c+s, n t+g) \vee(0,0)$ lies in $H$. If $n=0$ then $\operatorname{supp}(n c+s)=\operatorname{supp}(s)$ is finite, and $(s, g) \vee(0,0)=\left(s \vee 0, g^{\prime}\right)$, where $g^{\prime}=g \vee 0+\sum\left\{g_{\lambda}^{-}: \lambda \in\right.$ $\left.\operatorname{supp}\left(s^{+}\right)\right\}-\Sigma\left\{g_{\lambda}: \lambda \in \operatorname{supp}\left(s^{-}\right)\right\} \in G$. Here $g_{\lambda}$ designates the element of $S_{\lambda}$ such that $g-g_{\lambda} \in S_{\lambda}^{\perp}$. If $n>0$ then $\operatorname{supp}(n c+s)$ is cofinite, $\operatorname{supp}\left((n c+s)^{-}\right)$is finite, and

$$
(n c+s, n t+g) \vee(0,0)=\left((n c+s) \vee 0, g^{\prime}\right),
$$

where

$$
\begin{aligned}
g^{\prime}= & g-\sum\left\{\left(n t_{\lambda}+g_{\lambda}\right)^{-}: \lambda \notin \operatorname{supp}(n c+s)\right\} \\
& -\sum\left\{\left(n t_{\lambda}+g_{\lambda}\right): \lambda \in \operatorname{supp}\left((n c+s)^{-}\right)\right\} \in G .
\end{aligned}
$$

Here $t_{\lambda}$ is the unique element of $S_{\lambda}$ such that $S_{\lambda} \theta-t_{\lambda} \in S_{\lambda}^{\perp}$. The case in which $n<0$ is handled similarly.

Let $U=\{(u, 0): u \in \Sigma \mathbf{Z}\}$, an $l$-subgroup of $H$ which lies above $G$. To show that $U$ is maximal above $G$ consider $h=(n c+u, n t+g) \in H \backslash U$ and let $W$ be the l-subgroup of $H$ generated by $U \cup\{h\}$. Now for each $\lambda \in \Lambda$ there is an element $u_{\lambda} \in \sum \mathbf{Z}$ such that $(\lambda)\left(n c+u+u_{\lambda}\right)=0$. Therefore $n t_{\lambda}+g_{\lambda} \in G$ is a component of $h+\left(u_{\lambda}, 0\right) \in W$. Now if $n t_{\lambda}+g_{\lambda}=0$ for all $\lambda$, then $n t+g \in S_{\lambda}^{\perp}$ for all $\lambda$, hence $t-f \in S_{\lambda}^{\perp}$ for all $\lambda$, where $f$ is the element proven to exist in $G$ by Proposition 6.6 such that $n(-f)=g$. This contradicts the assumption that $\theta$ violates the SSP and proves that $n t_{\lambda}+g_{\lambda} \neq 0$ for some $\lambda \in \Lambda$. But then $\Gamma(W) \cap \Gamma(G) \neq \varnothing$, proving that $U$ is maximal above $G$. This proves the theorem since $U+G$ fails to contain $(c, t) \in H$, among others.

Suppose $P$ is a polar of the $l$-group $G$. We shall say that a set $X \subseteq G^{+}$is of type $\mathscr{W}(P)$ if $X$ is type $\mathscr{Y}, X \subseteq P^{+}$, and for every $0 \leqslant g \in G$ there is some element $g^{\prime} \in G$ satisfying the following three conditions: $g-g^{\prime} \in P, X \wedge g \leqslant g^{\prime}$, and $P \subseteq \mathrm{V}\left\{\left(g^{\prime}-x \wedge g\right)^{\perp}: x \in X\right\}$. We shall call $G$ sup $\mathscr{W}$ complete if $\mathrm{V} X$ exists in $G$ for every polar $P$ and every $X \subseteq G^{+}$of type $\mathscr{W}(P)$. Several comments deserve mention here. Though we shall not prove it, the element $g^{\prime}$ asserted to exist by the definition is unique for each $0<g \in G$, and in fact $0 \leqslant g^{\prime} \leqslant g$ in all cases. Also, not every type $\mathscr{W}(P)$ set $X$ is type $\mathscr{Z}$, nor is every type $\mathscr{Z}$ set $Y$ necessarily type $\mathscr{W}(P)$. For example, take $G=C[-1,1]$, the $l$-group of continuous real-valued functions on the interval $[-1,1]$, let $P=\{g \in G \mid \operatorname{supp}(g) \subseteq(0,1)\}$, let $X=\{g \in$ $\left.P^{+} \mid(r) g \leqslant 1 / r, r \in(0,1)\right\}$, and let $Y=\left\{g \in P^{+} \mid(r) g \leqslant r /(1-r), r \in(0,1)\right\}$. Nevertheless, the notions of type $\mathscr{Z}$ set and type $\mathscr{W}(P)$ set coincide for summands $P$.

Proposition 6.9. Suppose $P$ is a summand of the l-group $G$. $A$ set $X \subseteq P^{+}$is of type $\mathscr{W}(P)$ if and only if $X$ is type $\mathscr{Z}$ in $P$.

Proof. Suppose $X$ is of type $\mathscr{W}(P)$. Then $X$ is type $\mathscr{Y}$ in $G$ and therefore also in $P$. Consider $0<p \in P$, and let $p^{\prime}$ be the element provided by the definition of $X$ being type $\mathscr{W}(P)$. Then $p^{\prime} \in P$ because $p-p^{\prime} \in P, X \wedge p \leqslant p^{\prime}$, and

$$
\bigvee\left\{\left(p^{\prime}-x \wedge p\right)^{\perp}: x \in X\right\}=1_{\mathscr{Q}}
$$


where 2 is the polar algebra of $P$. By Lemma $6.1 \vee(X \wedge p)=p^{\prime} \in P$, meaning that $X$ is type $\mathscr{Z}$ in $P$.

Now suppose $X$ is type $\mathscr{Z}$ in $P$. Then $\vee\left\{((X-x) \vee 0)^{\perp}: x \in X\right\}=1_{\mathscr{Q}}$ and $X \subseteq P$ imply $\vee((X-x) \vee 0)^{\perp}=1_{\mathscr{P}}$, where $\mathscr{P}$ is the polar algebra of $G$, meaning $X$ is type $\mathscr{Y}$ in $G$. Given $0 \leqslant g \in G$ let $g=y+z$, where $y \in P$ and $z \in P \perp$, and let $g^{\prime}=w+z$, where $w=\mathrm{V}(X \wedge y)$ exists in $P$ because $X$ is type $\mathscr{Z}$ in $P$. Then $g-g^{\prime}=y-w \in P$, and $X \wedge g=X \wedge(y+z) \leqslant X \wedge y+z \leqslant w+z=g^{\prime}$. To show $P \subseteq \bigvee\left\{\left(g^{\prime}-x \wedge g\right)^{\perp}: x \in X\right\}$ in $\mathscr{P}$ first observe that $\left.\left(x_{1} \wedge y\right)-x \wedge y\right)^{+} \leqslant$ $\left(x_{1}-x\right)^{+}$for $x_{1}, x \in X$, from which follows $\bigvee\left\{((X \wedge y-x \wedge y) \vee 0)^{\perp}: x \in X\right\}$ $=1_{\mathscr{P}}$. A similar argument shows $\vee((-x \wedge y+X \wedge y) \vee 0)^{\perp}=1_{\mathscr{P}}$, and so $X \wedge y$ is type $\mathscr{Y}$. By Lemma $6.1 \vee(w-x \wedge y)^{\perp}=1_{\mathscr{P}}$, from which follows $\mathrm{V}\left(g^{\prime}-x \wedge g\right)^{\perp}$ $\supseteq P$.

Corollary 6.10. $X \subseteq G^{+}$is of type $\mathscr{W}(G)$ if and only if $X$ is type $\mathscr{Z}$.

Corollary 6.11. If $G$ is sup $\mathscr{W}$ complete, then every summand is sup $\mathscr{Z}$ complete. If $G$ is projectable and every polar is sup $\mathscr{Z}$ complete, then $G$ is sup $\mathscr{W}$ complete.

Lemma 6.12. Suppose $P$ is a polar of the l-group $G$, and that $X, Y \subseteq P^{+}$are sets of type $\mathscr{W}(P)$. Then $X+Y$ is of type $\mathscr{W}(P)$.

Proof. First we must show $X+Y$ to be type $\mathscr{Y}$. We know that $\mathrm{V} X=x_{0} \in G_{\mathscr{Y}}$ and $\bigvee Y=y_{0} \in G_{\mathscr{y}}$, hence by Lemma 6.1 and its analogue $\bigvee\left\{\left(y_{0}-y\right)^{\perp}: y \in Y\right\}=$ $\mathrm{V}\left\{\left(-x+x_{0}\right)^{\perp}: x \in X\right\}=1_{\mathscr{P} \text {. }}$ It follows that

$$
\begin{aligned}
1_{\mathscr{P}} & =\bigvee\left\{\left(\left(y_{0}-y\right)^{\perp} \cap\left(-x+x_{0}\right)^{\perp}\right): x \in X, y \in Y\right\} \\
& =\bigvee\left(\left(y_{0}-y\right) \vee\left(-x+x_{0}\right)\right)^{\perp}=\bigvee\left(\left(y_{0}-y\right)+\left(-x+x_{0}\right)\right)^{\perp} .
\end{aligned}
$$

Upon conjugation by $x_{0}$ we obtain $\mathrm{V}\left(\left(x_{0}+y_{0}\right)-(x+y)\right)^{\perp}=1_{\mathscr{P}}$, which proves $X+Y$ to be type $\mathscr{Y}$ in $G_{\mathscr{Y}}$ by Lemma 6.1. By Proposition 5.16 of [1], $X+Y$ is also type $\mathscr{Y}$ in the order dense $l$-subgroup $G$.

Now consider $0 \leqslant g \in G$ and let $g_{x}$ and $g_{y}$ be the elements of $G$ provided by the type $\mathscr{W}(P)$ property of $X$ and $Y$, respectively. Let $g^{\prime}=\left(g_{x}+g_{y}\right) \wedge g$. Then $0 \leqslant g-g^{\prime}=\left(g-g_{y}-g_{x}\right)^{+} \leqslant\left(g-g_{y}\right)^{+} \in P$, so $g-g^{\prime} \in P$. And for $x \in X$ and $y \in Y,(x+y) \wedge g \leqslant(x \wedge g)+(y \wedge g) \leqslant g_{x}+g_{y}$, which implies $(X+Y) \wedge g \leqslant$ $g^{\prime}$. Finally, to show $P \subseteq \mathrm{V}\left\{\left(g^{\prime}-(x+y) \wedge g\right)^{\perp}: x \in X, y \in Y\right\}$ consider $0_{\mathscr{P}} \neq T_{1}$ $\subseteq P$. Choose $x \in X$ such that $0_{\mathscr{P}} \neq\left(g_{x}-x \wedge g\right)^{\perp} \cap T_{1}=T_{2}$. The fact that $x \in P$ implies $0_{\mathscr{P}} \neq-x+T_{2}+x \subseteq P$, hence there must be some $y \in Y$ such that $0_{\mathscr{P}} \neq$ $\left(g_{y}-y \wedge g\right)^{\perp} \cap\left(-x+T_{2}+x\right)=T_{3}$. Note that $0_{\mathscr{P}} \neq x+T_{3}-x \subseteq T_{2}$. We claim $x+T_{3}-x \subseteq\left(g^{\prime}-(x+y) \wedge g\right)^{\perp}$. For if not then there is some $r \in x+T_{3}-x$ such that $0<r \leqslant g^{\prime}-(x+y) \wedge g$. Let $Q$ be a value of $r$, and observe that $Q+(x+y) \wedge g<Q+\left(g_{x}+g_{y}\right) \wedge g$. Since $r \in\left(g_{x}-x \wedge y\right)^{\perp} \backslash Q, Q+g_{x}=Q$ $+x \wedge y$. If $Q+g \leqslant Q+x$, then $Q+g_{x}=Q+g$ and $Q+(x+y) \wedge g=Q+g$ $=Q+\left(g_{x}+g_{y}\right) \wedge g$, a contradiction. Therefore $Q+g_{x}=Q+x<Q+g$. Now $-x+r+x \in\left(g_{y}-y \wedge g\right)^{\perp} \backslash R$ implies $R+g_{y}=R+y \wedge g$, where $R=-x+Q$ $+x$. If $R+y \leqslant R+g$ we get $R+g_{y}=R+y$, which gives the contradiction 
$Q+g_{x}+g_{y}=Q+x+y$. If $R+y>R+g$, then $R+g_{y}=R+g$, so $Q+x+y$ $>Q+x+g \geqslant Q+g$ and $Q+g_{x}+g_{y}=Q+x+g_{y}=Q+x+g \geqslant Q+g$, and we again have the contradiction $Q+(x+y) \wedge g=Q+g=Q+\left(g_{x}+g_{y}\right) \wedge$ g.

Theorem 6.14 is important and explains the significance of type $\mathscr{W}(P)$ subsets for our purposes. For its proof it is helpful to recast the definition of such sets slightly in Lemma 6.13, which in turn requires some terminology. Given an element $g$ and polar $P$ of $G$ we call $h$ the projection of $g$ on $P$ if $h \in P$ and $g-h \in P^{\perp} . h$ need not exist, but when it does we write $h$ as $g \nu P$. This terminology extends the use of $\nu$ in previous sections in the sense that $b v a$ there could also be written $b v[a]$ here.

Lemma 6.13. Suppose $X \subseteq G^{+}$and $P \in \mathscr{P}$. Then $X$ is of type $\mathscr{W}(P)$ if and only if there is an element $t \in G_{o y}$ satisfying the following conditions.

(a) $X \leqslant t \in P^{\perp \perp}$.

(b) $\bigvee\left\{(t-x)^{\perp}: x \in X\right\}=1_{\mathscr{P}}$.

(c) $(g-t)^{+} \nu P \in G$ for all $g \in G^{+}$.

Proof. Suppose $X$ is of type $\mathscr{W}(P)$; since $X$ is type $\mathscr{Y}$ there is some element $t \in G_{g}$ such that $\vee X=t$, and $t \in P^{\perp \perp}$ because $P^{\perp \perp}$ is a closed convex $l$-subgroup of $G_{\mathscr{y}}$. By Lemma 6.1, $\vee(t-x)^{\perp}=1_{\mathscr{P}}$. Given $g \in G^{+}$find $g^{\prime} \in G$ such that $g-g^{\prime} \in P$ and $P \subseteq \mathrm{V}\left(g^{\prime}-x \wedge y\right)^{\perp}$. We claim $g-g^{\prime}=(g-t)^{+} \nu P$, for which we must prove

$$
\begin{aligned}
(g-t)^{+}-\left(g-g^{\prime}\right) & =(g-t) \vee 0+g^{\prime}-g \\
& =\left(g^{\prime}-t\right) \vee\left(g^{\prime}-g\right)=g^{\prime}-t \wedge g \in P^{\perp} .
\end{aligned}
$$

If not then $P \cap\left(g^{\prime}-t \wedge g\right)^{\perp \perp}=R_{1} \neq 0_{\mathscr{P}}$, and so $\left(g^{\prime}-x_{1} \wedge g\right)^{\perp} \cap R_{1}=R_{2} \neq$ $0_{\mathscr{P}}$ for some $x_{1} \in X$, hence $\left(t-x_{2}\right)^{\perp} \cap R_{2} \neq 0_{\mathscr{P}}$ for some $x_{2} \in X$. Note that

$$
t \wedge g-x_{2} \wedge g=\left(\left(t-x_{2}\right) \wedge\left(g-x_{2}\right)\right) \vee((t-g) \wedge 0) \leqslant t-x_{2},
$$

so $\left(t \wedge g-x_{2} \wedge g\right)^{\perp} \cap R_{2} \neq 0$. By replacing $x_{1}$ and $x_{2}$ by $x=x_{1} \vee x_{2}$ we get $\left(g^{\prime}-x \wedge g\right)^{\perp} \cap(t \wedge g-x \wedge g)^{\perp} \cap\left(g^{\prime}-t \wedge g\right)^{\perp \perp} \neq 0_{\mathscr{P}}$. But this is a contradiction since

$$
\begin{aligned}
\left(g^{\prime}-x \wedge g\right)^{\perp} \cap(t \wedge g-x \wedge g)^{\perp} & \subseteq\left(g^{\prime}-x \wedge g-(t \wedge g-x \wedge g)\right)^{\perp} \\
& =\left(g^{\prime}-t \wedge g\right)^{\perp} .
\end{aligned}
$$

Conversely suppose $X$ and $t$ satisfy conditions (a), (b), and (c). Then $X$ is type $\mathscr{Y}$, $\vee X=t$ by Lemma 6.1 , and $X \subseteq P^{\perp \perp} \cap G=P$. Given $g \in G^{+}$let $g^{\prime}=$ $-(g-t)^{+} \nu P+g \in G$. Then $g-g^{\prime}=(g-t)^{+} \nu P \in P$. To show $X \wedge g \leqslant g^{\prime}$ consider an $x \in X$ and arbitrary prime $Q$ such that $Q<Q+x \wedge g$. Since $P \nsubseteq Q$ it follows that $(g-t)^{+} \nu P^{\perp} \in P^{\perp} \subseteq Q$, hence

$$
\begin{aligned}
Q+g^{\prime} & =Q-(g-t)^{+} \nu P+g=Q-(g-t)^{+}+g \\
& =Q=t \wedge g \geqslant Q+x+g .
\end{aligned}
$$

To show $P \subseteq \mathrm{V}\left\{\left(g^{\prime}-x \wedge g\right)^{\perp}: x \in X\right\}$ consider $0_{\mathscr{P}} \neq R \subseteq P$ and find $x \in X$ such that $(t-x)^{\perp} \cap R \neq 0_{\mathscr{g}}$. We claim $(t-x)^{\perp} \cap P \subseteq\left(g^{\prime}-x \wedge g\right)^{\perp} \cap P$. For if not there is some $0<p \in(t-x)^{\perp} \cap P$ such that $p \leqslant g^{\prime}-x \wedge g$. Let $Q$ be a 
value of $p$. Again $P \nsubseteq Q$ implies $(g-t)^{+} \nu P^{\perp} \in P^{\perp} \subseteq Q$, so

$$
\begin{aligned}
Q+x \wedge g & <Q+g^{\prime}=Q-(g-t)^{+} \nu P+g \\
& =Q-(g-t)^{+}+g=Q+t \wedge g .
\end{aligned}
$$

But $p \wedge(t-x)=0$ implies $t-x \in Q$ or $Q+t=Q+x$, hence $Q+x \wedge g=Q$ $+t \wedge g$, a contradiction.

If $X \subseteq G^{+}$is of type $\mathscr{W}(P)$ and $\bigvee X=t \in G_{\mathscr{O}}$, then $G$ need not be convex in the $l$-subgroup $\langle G \cup\{t\}\rangle \leqslant G_{g y}$. The following theorem asserts, however, that $t$ must be the projection on $P$ of an element $h$ in an extension $H$ in which $G$ is a convex $l$-subgroup. In the statement of this theorem $\mathrm{V}\left\{(h-x)^{\perp}: x \in X\right\}$ refers to the supremum in the Boolean algebra $\mathscr{P}$ of polars of $H$.

THEOREM 6.14. Suppose $G$ is an abelian l-group with polar $P$ and subset $X \subseteq G^{+}$. Then $X$ is of type $\mathscr{W}(P)$ if and only if there is an l-group $H$ and element $0<h \in H$ such that $G$ is a convex l-subgroup of $H, h \geqslant X \subseteq P$, and

$$
(G-H)^{+} \subseteq P \subseteq \bigvee\left\{(h-x)^{\perp}: x \in X\right\} .
$$

Proof. Suppose $G$ is a convex $l$-subgroup of an $l$-group $H$ as above. To prove that $X$ is of type $\mathscr{Y}$ consider $T \neq 0_{\mathscr{P}}$; we seek $x \in X$ such that $((X-x) \vee 0)^{\perp} \cap T$ $\neq 0_{\mathscr{P}}$. If $T \nsubseteq P$ we are done, for $((X-x) \vee 0)^{\perp} \supseteq P^{\perp}$ for every $x \in X$. Assuming $T \subseteq P$, find $x \in X$ such that $T \cap(h-x)^{\perp} \neq 0_{\mathscr{P}}$. Since $\left(x_{1}-x\right) \vee 0 \leqslant h-x$ for all $x_{1} \in X$, it follows that $((X-x) \vee 0)^{\perp} \cap T \neq 0_{\mathscr{P}}$. To prove that $X$ is of type $\mathscr{W}(P)$ consider an arbitrary $0 \leqslant g \in G$ and let $g^{\prime}=h \wedge g$, an element of $G$ by virtue of the convexity of $G$ in $H$. Then $g-g^{\prime}=(g-h)^{+} \in P$ by hypothesis, and $X \wedge g \leqslant h \wedge g=g^{\prime}$. To demonstrate that $P \subseteq \bigvee\left\{\left(g^{\prime}-x \wedge g\right)^{\perp}: x \in X\right\}$ consider $0_{\mathscr{P}} \neq T \subseteq P$ and find $x \in X$ such that $(h-x)^{\perp} \cap T \neq 0_{\mathscr{P}}$. Since

$$
0 \leqslant h \wedge g-x \wedge g=((h-g) \wedge(g-x)) \vee((h-g) \wedge 0) \leqslant h-x,
$$

it follows that $\left(g^{\prime}-x \wedge g\right)^{\perp} \cap T \neq 0_{\mathscr{P}}$.

Suppose $X$ is of type $\mathscr{W}(P)$. Let $K$ be $\mathbf{Z} \times \bar{G}$, the splitting extension of the orthocompletion $\bar{G}$ of $G$ by the integers $\mathbf{Z}$ determined by defining $\sigma(n)$ to be the identity $l$-automorphism on $\bar{G}$ and $\pi(n)=[n]=P^{\perp} \in \mathscr{S}(\bar{G})$ for all $0 \neq n \in \mathbf{Z}$. Let $\vee X=t \in \bar{G}$ and let $H$ be the subgroup $\{(n, n t+g): n \in \mathbf{Z}, g \in G\}$. We claim that $H$ is an $l$-subgroup of $K$, the proof of which requires showing $(n, n t+g) \vee$ $(0,0) \in H$ for all $n \in \mathbf{Z}$ and $g \in G$. Consider first the case in which $n>0$. By Lemma 3.9

$$
\begin{aligned}
(n, n t+g) \vee(0,0) & =\left(n,(n t+g) \nu n+(n t+g)^{+} \mu n\right) \\
& =\left(n, n t+g-(n t+g)^{-} \mu n\right) \\
& =\left(n, n t+g+(-g-n t)^{+} \mu n\right) \\
& =\left(n, n t+g+\left((-g)^{+}-n t\right)^{+} \nu P\right) .
\end{aligned}
$$

The point is that by Lemma $6.12 n X=\left\{\sum_{i=1}^{n} x_{i}: x_{i} \in X\right\}$ is type $\mathscr{W}(P)$ with supremum $n t$, and $\left((-g)^{+}-n t\right)^{+} \nu P$ exists in $G$ by Lemma 6.13. Therefore $(n, n t+$ $g) \vee(0,0) \in H$ in this case. In case $n=0$ we have

$$
(0, g) \vee(0,0)=(0, g \vee 0) \in H,
$$


and in case $n<0$ we have

$$
\begin{aligned}
(n, n t+g) \vee(0,0) & =\left(0,(n t+g)^{+} \mu n\right)=\left(0,(n t+g)^{+} \nu P\right) \\
& =\left(0,\left(g^{+}-(-n) t\right)^{+} \nu P\right) .
\end{aligned}
$$

As before, $k X=\left\{\sum_{i=1}^{k} x_{i}: x_{i} \in X\right\}$ is type $\mathscr{W}(P)$ with supremum $k t$, where $k=-n$, and so $\left(g^{+}-k t\right) \nu P \in G$ by Lemma 6.13. This completes the proof that $H$ is an $l$-subgroup of $K$, and $G$ is clearly convex in $H$.

Let $h=(1, t) \in H$; we must verify that $(G-h)^{+} \subseteq P \subseteq \bigvee\left\{(h-x)^{\perp}: x \in X\right\}$. Now for any $g \in G$,

$$
(g-h)^{+}=(-1, g-t) \vee(0,0)=\left(0,(g-t)^{+} \nu P\right)=\left(0,\left(g^{+}-t\right)^{+} \nu P\right) \in P .
$$

To show $P \subseteq \mathrm{V}(h-x)^{\perp}$ consider $0_{\mathscr{P}} \neq R \subseteq P$, then find $x \in X$ such that $(t-x)^{\perp} \cap R \neq 0_{\mathscr{P}}$. For $0<p \in R \cap(t-x)^{\perp}$ we have

$$
p \wedge(h-x)=(0, p) \wedge(1, t-x)=(0,(t-x) \wedge p)=(0,0) \text {. }
$$

This completes the proof.

Proposition 6.15. An abelian l-group $G$ which splits off from below in every abelian l-group in which it is a convex l-subgroup must be sup $\mathscr{W}$ complete. In this case any polar $P$ which admits a type $\mathscr{W}(P)$ subset is a cardinal summand.

Proof. Suppose $P$ is a polar and $X$ a type $\mathscr{W}(P)$ subset of $G$ and construct $H$ with element $h$ as in the proof of Theorem 6.14. Suppose for contradiction that $\mathrm{V} X=t \in \bar{G} \backslash G$. We claim $\{0\}$ is maximal above $G$. Now an arbitrary element of $H^{+} \backslash G$ has the form $n h+g=(n, n t+g)$ for $0<n \in \mathbf{Z}$ and $g \in G$. Note that $(n t+g) \nu P=n t+g \nu P \neq 0$ in $\bar{G}$, for otherwise $0<t \leqslant n t \leqslant|g|$ implies $t \in G$. By Proposition $3.15(n, n t+g)$ has a value in $\Gamma(G)$, hence $G$ does not lie below $\langle(n, n t+g)\rangle$. This proves the claim, and since the claim directly violates the hypothesis of the proposition, we can only conclude that $t \in G$, or that $G$ is sup $\mathscr{W}$ complete.

Observe that $h-t=(1,0)$ lies above $G$, and that $H(h-t) \cap G=P^{\perp}$, so $P \in \mathscr{S}(G)$ by Proposition 1.1 .

We have arrived at last at the main theorem.

THEOREM 6.16. An abelian l-group $G$ splits off from below in every abelian l-group in which it is a convex l-subgroup if and only if $G$ is divisible, sup $\mathscr{W}$ complete, and has the SSP.

Proof. Suppose $G$ has the three listed properties and is a convex $l$-subgroup of the abelian $l$-group $H$. Let $U$ be an $l$-subgroup of $H$ maximal above $G$, and consider an arbitrary $0<h \in H$. We shall produce an element $g \in G$ such that $h-g \in U$. All polar computations will be done in $\mathscr{P}$, the Boolean algebra of polars of $H$.

For each $u \in U$ and positive integer $n$ let

$$
\begin{gathered}
P(n, u)=P=\bigvee\left\{((g-|n h-u|) \vee 0)^{\perp \perp}: g \in G^{+}\right\}, \\
X_{1}=P^{+} \wedge(n h-u)^{+}, \quad \text { and } X_{2}=P^{+} \wedge(u-n h)^{+} .
\end{gathered}
$$


We claim $P \subseteq \bigvee\left\{\left((n h-u)^{+}-x_{1}\right)^{\perp}: x_{1} \in X_{1}\right\}$. To verify this consider $0_{\mathscr{P}} \neq T \subseteq P$ and find $0<g \in G$ such that $((g-|n h-u|) \vee 0)^{\perp \perp} \cap T \neq 0_{\mathscr{P}}$, then find $0<t \in T$ with $t \leqslant(g-|n h-u|)^{+} \leqslant\left(g-(n h-u)^{+}\right)^{+}$. Let $x_{1}=g \wedge(n h-u)^{+} \in X_{1}$. Then

$$
t \wedge\left((n h-u)^{+}-x_{1}\right)=t \wedge\left((n h-u)^{+}-g\right)^{+}=0,
$$

proving the claim. By Theorem $6.14 X_{1}$, and analogously $X_{2}$, are of type $\mathscr{W}(P)$, so by Proposition 6.15 there are elements $a, b \in G$ with $\bigvee X_{1}=a$ and $\bigvee X_{2}=b$, and $P(n, u)$ is a summand. By the divisibility of $G$ there is some $g(n, u) \in G$ such that $n g(n, u)=a-b$.

We claim $n(h-g(n, u))-u$ lies above $G$. To see this, first observe that $(n h-u)^{+}-a \in P^{\perp}$ and $-(n h-u)^{-}-b \in P^{\perp}$, hence $n(h-g(n, u))-u \in P^{\perp}$. If there were some prime $Q$ and element $g \in G$ such that $Q<Q+\mid n(h-g(n, u))$ $-u \mid<Q+g$, then

$$
Q+|n h-u|-|n g(n, u)| \leqslant Q+|n(h-g(n, u))-u|<Q+g,
$$

so $Q+|n h-u|<Q+g+|n g(n, u)|$, meaning $((g+|n g(n, u)|)-|n h-u|) \vee 0$ is an element of $P \backslash Q$. Therefore $n h-u-n g(n, u) \in P^{\perp} \subseteq Q$, contradicting $Q<Q$ $+|n(h-g(n, u))-u|$.

We claim the map $\theta$ which takes each $P(n, u)$ to $g(n, u)$ is consistent. That is, $g(n, u)-g(m, v) \in[P(n, u) \cap P(m, v)]^{\perp}$ for all $u, v \in U$ and positive integers $m$ and $n$. For if not there is some prime $Q$ of $G$ such that $Q+g(n, u) \neq Q+g(m, v)$ and $Q \nsupseteq P(n, u) \cap P(m, v)$. We have seen in the previous paragraph that $n(h-g(n, u))-u$ and $m(h-g(m, v))-v$ lie in $P^{\perp}$. Therefore $\bar{Q}+n h-u=\bar{Q}$ $+n g(n, u)$ and $\bar{Q}+m h-v=\bar{Q}+m g(m, v)$, where $\bar{Q}$ is the unique prime subgroup of $H$ whose intersection with $G$ is $Q$. Thus

$$
\begin{aligned}
\bar{Q}+m n(g(m, v)-g(n, u)) & =\bar{Q}+((m n h-n v)-(m n h-m u)) \\
& =\bar{Q}+(m u-n v) .
\end{aligned}
$$

It cannot be that $m u-n v \in U$ fails to lie in $\bar{Q}$, since in this case the value of $m u-n v$ containing $\bar{Q}$ would correspond to the value of $m n(g(m, v)-g(n, u))$ containing $Q$, and $m u-n v$ would have a value in $\Gamma(G)$ contrary to the assumption that $U$ lies above $G$. But then $\bar{Q}+g(n, u)=\bar{Q}+g(m, v)$, contrary to the assumption that $Q+g(n, u) \neq Q+g(m, v)$. This completes the proof that $\theta$ is consistent.

Let $g \in G$ result from applying the SSP to $\theta$; explicitly, $g-g(n, u) \in P(n, u)^{\perp}$ for all $u$ and all $n$. If $h-g \notin U$, then by Proposition 1.9 there is some $u \in U$ and positive integer $n$ such that $n(h-g)-u$ has value $Q$ in $\Gamma(G)$. Let $0<k \in G$ satisfy $Q+k>Q+|n(h-g)-u|$. Since $Q+(k+n g)>Q+(n h+u)$ and $Q$ $+(k-n g)>Q+(u-n h)$ it follows that $Q+(k+|n g|)>Q+|n h-u|$, that is $(k+|n g|-|n h-u|)^{+} \in P(n, u) \backslash Q$. Since $Q \nsupseteq P(n, u), g-g(n, u) \in P(n, u)^{\perp}$ $\subseteq R$ for all prime subgroups $R \subseteq Q$. Therefore

$$
R+(n(h-g)-u)=R+(n(h-g(n, u)-u))
$$

for all prime subgroups $R \subseteq Q$, and in particular, $n(h-g(n, u))-u$ has value $Q \in \Gamma(G)$. This contradicts the fact that $n(h-g(n, u))-u$ lies above $G$. 
COROLlaRY 6.17. A divisible abelian l-group which is sup $\mathscr{Y}$ complete splits off from below in every abelian l-group in which it is a convex l-subgroup.

THEOREM 6.18. Suppose $G$ is a divisible abelian sup $\mathscr{Y}$ complete l-group. Then a convex $l$-subgroup $L$ splits off from below in $G$ if and only if $L$ is maximal below if and only if $L$ is closed.

Proof. Given a closed convex $l$-subgroup $L$, we observe that each type $\mathscr{Y}$ subset $X \subseteq L^{+}$remains type $\mathscr{Y}$ in $G$. Therefore $L$ is sup $\mathscr{Y}$ complete and divisible.

COROLlaRY 6.19. The maximal below l-subgroups of a divisible abelian sup 9 complete l-group form a lattice under the inclusion order.

Corollary 6.20. Suppose $G$ is a divisible abelian sup $\mathscr{Y}$ complete l-group, and that $U_{1}$ and $U_{2}$ are both maximal above the convex l-subgroup $L$. Then there is an l-automorphism $\theta$ of $G$ such that $\theta$ is the identity on the closure of $L$ and $U_{1} \theta=U_{2}$.

LEMMA 6.21. Suppose $G$ is an abelian l-group. If $G$ is divisible, then so is $G_{\mathscr{O} \text {. }}$

Proof. Given $X$ a type $\mathscr{Y}$ subset of $G$ and positive integer $n$ let $Y=\{z \in G \mid n z$ $\in X$ \}. Then $Y$ must be type $\mathscr{Y}$, for if $x_{1}, x_{2} \in X$ and if $n y_{i}=x_{i}$, then

$$
\left(\left(x_{1}-x_{2}\right) \vee 0\right)^{\perp}=\left(\left(n y_{1}-n y_{2}\right) \vee 0\right)^{\perp}=\left(n\left(y_{1}-y_{2}\right) \vee 0\right)^{\perp}=\left(\left(y_{1}-y\right) \vee 0\right)^{\perp},
$$

meaning $\left(\left(X-x_{i}\right) \vee 0\right)^{\perp}=\left(\left(Y-y_{i}\right) \vee 0\right)^{\perp}$. To prove the lemma form $G_{\mathscr{g}}$ inductively as follows. Let $G_{0}=G$, and if $G_{\alpha}=G_{\mathscr{y}}$ let $G_{\alpha+1}=G_{\alpha}$. If $G_{\alpha} \neq G_{\mathscr{y}}$ choose $0<x \in G_{\mathscr{y}} \backslash G_{\alpha}$ and let $G_{\alpha+1}$ be the $l$-subgroup of $G_{\mathscr{y}}$ generated by $G_{\alpha} \cup\{q x: q$ rational $\}$. Let $G_{\beta}=\cup_{\alpha<\beta} G_{\alpha}$ for limit ordinals $\beta$. A simple induction shows $G_{\alpha}$ divisible for all $\alpha$, hence $G_{o y}$, which is $G_{\alpha}$ for sufficiently large ordinals $\alpha$, is also divisible.

In the next proposition $G^{d}$ designates the $l$-group divisible hull of $G$.

Proposition 6.22. Any abelian l-group $G$ is a large l-subgroup of some abelian l-group $H$ which splits off from below in every abelian l-group in which it $(H)$ is a convex l-subgroup. $H$ may be taken to be $\left(G^{d}\right)_{y}$.

We close this section by giving an example of an l-group $G$ which is sup $\mathscr{W}$ complete but lacks the SSP. Though $G$ is simple in structure in the sense that $\sum_{n=1}^{\infty} \mathbf{Z}_{n} \leqslant G \leqslant \prod_{n=1}^{\infty} \mathbf{Z}_{n}$, the construction itself uses the Continuum Hypothesis: let $\left\{f_{\alpha}: \alpha<\aleph_{1}\right\}$ be an enumeration of all functions from $N$ into $N$, where $N=$ $\{1,2,3 \ldots\}$. Define $G_{0}=\sum_{n=1}^{\infty} \mathbf{Z}_{n}$. Given countable $G_{\alpha}$ define $G_{\alpha+1}$ as follows. Let $\left\{w_{k}(x): k \in N\right\}$ be an enumeration of all l-group terms of the form $\bigvee_{i=1}^{n} \bigwedge_{j=1}^{m}\left(g_{i j}+n_{i j} x\right)$, where $g_{i j} \in G_{\alpha}, n_{i j} \in \mathbf{Z}$, and $x$ is an indeterminate. Iteratively define a function $f: N \rightarrow N$ such that $(n) f \geqslant(n) f_{\alpha}$ for all $n \in N$, and such that $(n)\left(w_{k}(f)\right) \neq 1$ for all $1 \leqslant k \leqslant n \in N$. Let $G_{\alpha+1}$ be the $l$-subgroup of $\prod_{n=1}^{\infty} \mathbf{Z}_{n}$ generated by $G_{\alpha} \cup\{f\}$. Define $G_{\gamma}=\bigcup\left\{G_{\alpha}: \alpha<\gamma\right\}$ for limit ordinals $\gamma \leqslant \boldsymbol{\aleph}_{1}$. Let $G$ be the projectable hull of $G_{\aleph_{1}}$. That is, $G$ consists of those elements $g \in \Pi \mathbf{Z}_{n}$ for which there exist disjoint sets $X_{1}, X_{2}, \ldots, X_{n} \subseteq N$ such that $\bigcup_{i=1}^{n} X_{i}=N$, and such that for each $X_{i}$ there is some $g_{i} \in G_{\aleph_{1}}$ with $(n) g=(n) g_{i}$ for all $n \in X_{i}$. Observe 
that for any $g \in G\{n \in N:(n) g=1\}$ is finite since this condition holds for all $g \in G_{\aleph_{1}}$. Since $\sum \mathbf{Z}_{n} \leqslant G \leqslant \Pi \mathbf{Z}_{n}$ and since every polar of $G$ is a cardinal summand, $G$ cannot possess the SSP.

To demonstrate that $G$ is sup $\mathscr{W}$ complete we need only show that every polar is sup $\mathscr{Z}$ complete. Consider a type $\mathscr{Z}$ subset $X$ of polar $P$. Because $X$ is type $\mathscr{Y}$, $\{(n) p: p \in X\}$ is a bounded set for each index $n \in N$. Let $\alpha<\aleph_{1}$ be such that $(n) f_{\alpha}=\bigvee\{(n) p: p \in X\}$ for each $n \in N$. There is by construction some element $g \in G$ with $g \geqslant f_{\alpha}$, and by projecting it on $P$ we may assume $g \in P$. By definition of type $\mathscr{Z}, \mathrm{V} X=\mathrm{V}(X \wedge g)$ exists in $P$. We have shown $P$ to be sup $\mathscr{Z}$ complete, and hence $G$ to be sup $\mathscr{W}$ complete.

\section{REFERENCES}

1. R. N. Ball, Convergence and Cauchy structures on lattice ordered groups, Trans. Amer. Math. Soc. 259 (1980), 357-392.

2. The generalized orthocompletion and strongly projectable hull of a lattice ordered group, Canad. J. Math. 34 (1982), 621-661.

3. _ The structure of the $\alpha$-completion of a lattice ordered group, Pacific J. Math. (submitted).

4. __ Topological lattice ordered groups, Pacific J. Math. 83 (1979), 1-26.

5. A. Bigard, Contribution à la théorie des groupes réticulés, Thèse sci. math., Paris, 1969.

6. A. Bigard, K. Keimel and S. Wolfenstein, Groupes et anneaux réticules, Lecture Notes in Math., vol. 608, Springer-Verlag, Berlin and New York, 1977.

7. J. W. Brewer, P. Conrad and P. Montgomery, Lattice ordered groups and a conjecture for adequate domains, Proc. Amer. Math. Soc. 43 (1974), 31-35.

8. R. D. Byrd, Lattice ordered groups, Thesis, Tulane University, 1966.

9. P. Conrad, Lattice ordered groups, Lecture Notes, Tulane University, 1970.

10. _ Lex subgroups of lattice ordered groups, Czechoslovak Math. J. 18 (1968), 86-103.

11. The structure of an l-group that is determined by its minimal prime subgroups, Ordered Groups, Lecture Notes in Pure and Appl. Math., vol. 62, Dekker, New York, 1980.

12. P. Conrad, J. Harvey and C. Holland, The Hahn embedding theorem for lattice ordered groups, Trans. Amer. Math. Soc. 108 (1963), 143-169.

13. A. Glass, Ordered permutation groups, London Math. Soc. Lecture Notes Series 55, Cambridge Univ. Press, London, 1981.

14. S. McCleary, Closed cls of a normal valued l-group ...

15. E. Scrimger, A large class of small varieties of lattice ordered groups, Proc. Amer. Math. Soc. 51 (1975), 301-306.

Department of Mathematics, Boise State University, Boise, IDaho 83725

Department of Mathematics, University of Kansas, Lawrence, Kansas 66045

Department of Mathematics, INDiana University, SOUTH BeND, INDiana 46634 